
\title{
Research Suare \\ Screening and Joint Analysis of Key IncRNAs for Milk Fat Metabolism in Dairy Cows
}

\section{Tong Mu}

Ningxia University

\section{Honghong $\mathrm{Hu}$}

Ningxia University

Xiaofang Feng

Ningxia University

\section{Yanfen Ma}

Key Laboratory of Ruminant Molecular and Cellular Breeding, Ningxia Hui Autonomous Region, Ningxia University, Yinchuan 750021, China.

\section{Ying Wang}

Ningxia University

Jiamin Liu

Ningxia University

\section{Baojun Yu}

Ningxia University

\section{Wan Wen}

Animal Husbandry Extension Station, Yinchuan 750001, China

\section{Juan Zhang}

Ningxia University

Yaling Gu ( $\nabla$ guyl@nxu.edu.cn )

Ningxia University

\section{Research Article}

Keywords: Holstein cattle, IncRNAs, gene, joint analysis, MFP

Posted Date: May 24th, 2021

DOI: https://doi.org/10.21203/rs.3.rs-495669/v1

License: (c) (i) This work is licensed under a Creative Commons Attribution 4.0 International License. Read Full License 


\section{Abstract}

Background: Long noncoding RNAs (IncRNAs) play an important regulatory role in various biological processes as a key regulatory factor. However, there are largely unknown for the function and expression profile of IncRNAs in milk fat synthesis of dairy cows.

Results: In this study, RNA sequencing (RNA-seq) was used to research the whole genome expression of IncRNAs and mRNA transcripts in bovine mammary epithelial cells (BMECs) of dairy cows with high and low milk fat percentage (MFP), and joint analysis was carried out. We identified a total of 47 differentially expressed genes (DEGs) and 38 differentially expressed IncRNAs (DELs, Padj < 0.05), 11 candidate DEGs that may regulate milk fat metabolism were screened by enrichment analysis. Downregulated differential gene ENPP2 and upregulated differential gene BCAT1 are more likely to participate in the milk fat metabolism, and its function needs further experiments verification. The enrichment analysis of target genes predicted by DELs identified 7 cis (co-localization) and 10 trans (co-expression) candidate target genes related to milk lipid metabolism, corresponding to a total of 18 DELs. Among them, the targeting relationship between long intervening/intergenic noncoding RNA (lincRNA) TCONS_00082721 and FABP4 gene that predicts milk fat metabolism by co-localization and co-expression is worthy of attention. Based on the expression information of DELs, differential microRNAs (miRNAs), and lipid metabolism-related target genes, 156 competing endogenous RNAs (ceRNAs) interaction regulation networks related to milk fat metabolism were constructed. The regulatory network centered on miR-145 will be the focus of subsequent experimental research. The ceRNAs regulatory network related to TCONS_00082721 and TCONS_00172817 are more likely to be involved in milk fat synthesis.

Conclusions: These results will provide new ways to understand the complex biology of dairy cow milk fat synthesis and provide valuable information for the breed improvement of Chinese Holstein cattle.

\section{Background}

Milk quality is affected by many factors, such as population genetic structure, reproductive performance, feeding and management level, and is closely related to the main raw material composition of milk. Milk fat is an important component in butter and yogurt, and its content and composition are the main reference elements in milk quality evaluation. Nowadays, milk fat content is not only one of the important indicators of core competitiveness of dairy industry, but also the main target feature of dairy cow breeding [1]. To a certain extent, it also plays an important role in nutrition and metabolism during human growth and development [2]. Therefore, the exploration of the theory and ways of milk fat formation and regulation, and the improvement of milk fat content have become the focus of lactation biology research in the world.

In the past, many scholars have extensively studied on the complex regulatory mechanisms of breast development and elucidated the main ways of milk fat synthesis (including ab initio synthesis and absorption of FA in the blood) [3]. Quantitative real-time reverse transcription PCR (qRT-PCR) can better 
discover the transcriptional regulation steps of milk fat synthesis. High-throughput sequencing has identified a series of effective genes and regulatory factors in milk fat metabolism [4]. As we all know, the study of gene regulation mainly depends on protein-coding genes. However, more and more evidence suggests that non-coding RNAs (ncRNAs), which are mistaken for "transcriptional noise", are also important factors in regulating complex developmental processes in organisms [5]. Long noncoding RNAs (IncRNAs) are newly identified long-stranded non-coding RNAs in the breast, with a length of more than 200 nucleotides and more tissue-specific than coding genes [6]. The mode of action of IncRNAs on genes is more complex than that of microRNAs (miRNAs). At present, transcriptional interference is a more mature regulatory model [7]. Functional studies in mammals have found that IncRNAs regulate gene expression at epigenetic, transcriptional and post-transcriptional levels, and also play key regulatory roles in a variety of biological processes [8]. In regulating milk fat metabolism in goats, Yu et al. [9] found that compared with early lactation, the expression trend of IncRNAs TCONS-00144434, TCONS-00148514 and TCONS-00055666 during mature lactation was the same as that of FASN, LPL, GPAM and MSMO1 genes with the function of fatty acid biosynthesis and cholesterol storage (The full names of all genes in this paper are shown in Supplementary Table 1). However, the specific regulatory mechanism remains to be further studied. Chao et al. [10] found that the target genes of TCONS_00162862 in dairy cow were involved in lipid transporter protein activity, ligase activity, and fatty acid transport, and mainly enriched in MAPK, PI3K-Akt, and insulin signaling pathways. Zheng et al. [11] performed transcriptome sequencing on four breast tissues of Holstein cows during peak lactation and late lactation, and found that the target genes of differentially expressed LNC-XLOC_274111 were FABP3 and FABP4, which would encode fatty acid-binding proteins in the bovine mammary gland. The target genes of XLOC_000752 were significantly enriched in PPAR, AMPK, and insulin signaling pathways, as well as glycerol metabolism pathways.

So far, research on IncRNAs in milk fat metabolism of ruminants are relatively lacking. The functions and expression profiles of IncRNAs in the bovine mammary gland are still unclear, and there are even fewer studies to screen differentially expressed IncRNAs (DELs) for differences in high and low milk fat percentage (MFP). In addition, the dynamic pattern of the interaction of ceRNA in milk fat synthesis remains unknown. To better understand the metabolic process of milk fat synthesis during lactation, Holstein cows with extreme differences in MFP were screened, fresh milk was collected and bovine mammary epithelial cells (BMECs) were isolated. The differentially expressed genes (DEGs) and DELs related to milk fat synthesis in the same lactation stage were screened by transcriptomics technology, and the prediction of IncRNAs targeting miRNAs to construct a ceRNA regulatory network of genes, miRNAs and IncRNAs related to milk fat metabolism, It is an important step in understanding the complex biological processes involved in milk production, and provides valuable information for the improvement of Chinese Holstein cattle breeds.

\section{Results}

\section{Isolation, culture, and identification of BMECs from milk}


As shown in Fig.1, the fresh milk (per $40 \mathrm{~mL}$ ) of dairy cows with high MFP and low MFP was centrifuged at $1000 \mathrm{r} / \mathrm{min}$ for $10 \mathrm{~min}$. It is found that the milk fat layer area of high MFP dairy cows are higher than that of low MFP dairy cows, and there are also different milk fat layer thickness $(a, b)$. The isolated BMECs is a unique "pebble" epithelial cells shape, and c-f is the flow chart of the isolation BMECs, genetics, growth, and cell secretory characteristics are normal [12]. The cell growth curve conforms to the "S" rule, indicating that the isolated cells have grown well [13]. Keratin 18 belongs to the intermediate fibrin family, is participated in the formation of the cytoskeleton, and is the marker of epithelial cells [13]. In this study, immunofluorescence showed that keratin 18 was positive $(\mathrm{g}, \mathrm{h})$, and the expression of keratin 8 (Epithelial cell-specific protein) was detected in both high and low groups (Fig. 2a), indicating that the isolated cells had specific epithelial cell characteristics [12]. Chromosome analysis showed that the cells were in a normal diploid configuration and contained 60 chromosomes, which was consistent with the chromosome number of dairy cows (i).

\section{BMECs TAG content and expression level of adipogenic genes in high and low milk fat groups}

Shen et al. [14] showed that the isolated BMECs maintained cell-specific genetic, structural, and biological functions in the $2^{\text {nd }}$ and $5^{\text {th }}$ passages. To confirm whether the BMECs isolated from the high-milk fat group and low-milk fat group have the differences in milk fat synthesis at the cellular level, we detected the changes in the TAG content and adipogenic gene expression of F2 generation BMECs. The results found that there was a great difference in TAG content, and the expression levels of fat-related genes $S C D, P P A R Y$, and FABP3 were higher in high-milk fat group than those in low-milk fat group, which further confirmed the difference between high and low MFP groups at the molecular level. It has laid a reliable material foundation for subsequent transcriptome sequencing and other experiments (Fig. 2).

\section{Overview of BMECs sequencing data}

Using Illumina PE150 sequencing platform, 81605996 - 97102888 and 78710246 - 88676080 raw_reads were obtained in high and low MFP BMECs, and 80633532 - 94731948 and 76807276 - 86 508476 clean_reads were obtained after removing the adapter related, containing $N$ and low quality, respectively. The sequencing error rate of the 8 samples is $0.02 \%$, Q20 is greater than $97 \%$, Q30 is greater than $94 \%$, the GC content is about $53 \%$, which ensures the accuracy of the subsequent analysis. The genomic distribution of clean_reads showed that most of the clean_reads were located in the exon region $(61.79 \%-70.27 \%)$, the intergenic region $(20.96 \%-24.41 \%)$, and the intron region $(8.03 \%-14.46 \%)$ of the bovine genome.

CPC/PFAM/CNCl software predicted that there were 25702 novel_mRNA with coding potential and 7602 candidate novel_IncRNAs without coding potential. Among all the candidate novel_IncRNAs, the IncRNAs located in the intergenic region account for $51.8 \%$, the antisense contains $24.75 \%$, the sense overlapping 
contains $23.50 \%$, and no sense intronic. After obtaining the FPKM of all transcripts, the distribution of transcripts expression levels of different samples was shown by block diagram (Fig. 3a), indicating that the BMECs expression level in cows with high MFP was slightly higher than that in cows with low MFP. The correlation of gene expression level between samples is an important index for testing the experimental reliability and sample selection. In this study, the correlation coefficients of the intra-group and inter-group samples were calculated based on the FPKM values of all genes in each sample and drawn into a heat map (Fig. 3b). As can be seen from the figure, intra-group correlation coefficients are all above 0.96 , graeter than the ideal sampling and experimental conditions $\left(R^{2}=0.92\right)$, indicating a high degree of similarity in expression patterns among samples and a difference between groups. Therefore, we think that the transcriptome sequencing results are reliable and can be used for subsequent analysis.

\section{Differential expression and clustering analysis of DEGs and DELs}

Among all the differential expressed mRNAs screened, a total of 15573 mRNAs were found in the highmilk fat group and the low-milk fat group, 717 in the high-milk fat group, and 614 in the low-milk fat group, However, the number of IncRNAs shared between the high-milk fat group and the low-milk fat group was relatively small (4 666), with 372 specific IncRNAs in the high-milk fat group and 365 IncRNAs in the low-milk fat group. A total of 47 significant DEGs and 38 DELs were screened from BMECs with high and low MFP. The FPKM of DEGs and DELs were transformed by $\log _{10}(F P K M+1)$ and then clustered by heat map (Fig. 4). DEGs or DELs with similar expression patterns cluster together and differ significantly in color, indicating that these genes share a common function or are involved in a common metabolic pathways. Compared with the low-milk fat group, 24/27 DEGs/DELs expression in the highmilk fat group was significantly upregulated, and 23/11 DEGs/DELs expression was significantly downregulated.

\section{DEGs enrichment analysis and screening of DEGs related to lipid metabolism}

Based on the functional enrichment analysis of 47 DEGs, a total of 173 significantly enriched GO items were identified, as shown in Fig. 5a, showing the first 20 significantly enriched GO items (the same below). Among the significantly enriched $\mathrm{GO}$ items, the BP related to lipid metabolism is the regulation of phospholipid translocation, positive regulation of phospholipid translocation, and glycerophospholipid catabolic process. The MF item is triglyceride lipase activity, lipase activity, aminophospholipid transporter activity, phosphodiesterase I activity, sterol esterase activity, and carboxylic ester hydrolase activity. KEGG enrichment analysis showed that 6 signaling pathways were significantly enriched (Fig. $5 b)$, and the pathways related to lipid metabolism were Wnt, Rap1 and 2-0xocarboxylic acid metabolism.

11 DEGs that may regulate milk fat metabolism were screened out (Table 1). The enrichment of GO/KEGG is shown in Supplementary Table 2. PDGFD, BCAT1, and APOL3 genes expression was 
upregulated, while ATP8A2, PTPRR, KCNMA1, ZFYVE28, ENPP2, DKK1, CES4A, and CTSH genes expression was downregulated. ENPP2 gene is significantly enriched to BP, MF, and KEGG pathway related to lipid metabolism, and it is inferred that it is more likely to regulate milk fat metabolism.

\section{Target genes prediction and functional analysis of DELs}

The target genes (cis) of DELs predicted by co-localization were enriched to 258 significant GO items $(P<$ 0.05), as shown in Fig. 6a. In the significantly enriched GO items, the BP is related to lipid metabolism that regulates the secretion of arachidonic acid, and MF is lipid phosphatase activity. KEGG enrichment analysis showed that 7 signal pathways were significantly enriched (Fig. 6b), among which Fc gamma Rmediated phagocytosis was related to lipid metabolism. Through enrichment analysis, 7 candidate target genes that may regulate milk fat metabolism were screened, namely VARS2, ITGA6, ATP4A, PPAP2C, FGF1, AMPH, and SYK. The enrichment of GO/KEGG is shown in Supplementary Table 3.

The co-expression predicted DELs target genes (trans) were significantly enriched to $509 \mathrm{GO}$ items $(P<$ 0.05), as shown in Fig. 7a. Among them, BP related to lipid metabolism includes lipid metabolic process, long-chain fatty-acyl-CoA biosynthetic process, fatty acid extension, fatty acid transport and negative regulation of triglyceride biosynthetic process, and so on. MF items includes fatty acid synthase activity, fatty acid transporter activity, long-chain fatty acid-binding, and lysophospholipid transporter activity, and so on. The target genes were significantly enriched to 17 signal pathways $(P<0.05)$ (Fig. $7 \mathrm{~b})$, among which the pathways related to lipid metabolism include PPAR, CAMP, Fatty acid metabolism and Fc epsilon RI. Under the condition that the correlation coefficient between DELs and target gene expression was greater than $0.97,10$ target genes that might regulate milk fat metabolism were screened out, namely PLA2G4E, INPP4B, FABP3, ACADSB, FABP4, OXSM, FABP7, GPX1, CYP27A1, and ALOX12 genes. The GO/KEGG enrichment is shown in Supplementary Table 4. Among the 10 lipid metabolism-related target genes, the expression of PLA2G4E, FABP4, FABP3, and ACADSB genes was upregulated $(P<0.05)$, while the expression of $F A B P 7$ and $C Y P 27 A 1$ genes was downregulated $(P<0.05)$. The upregulation of PLA2G4E, FABP4 and OXSM genes is significantly enriched in lipid metabolism-related BP, MF and KEGG. Therefore, these three genes and their corresponding DELs need more attention in the follow-up functional mechanism research.

The 17 lipid metabolism-related target genes were predicted and screened by co-localization and coexpression. All DELs that regulate these target genes are shown in Table 2. A total of 18 DELs that may be related to milk fat metabolism were screened and their corresponding relationships with target genes are shown in Supplementary Tables 5 and 6 . Among the 18 DELs that may be related to milk fat metabolism, 12 were upregulated and 6 were downregulated. Bold font is the DELs predicted by both colocalization and co-expression that may regulate milk fat metabolism. Italic is the DELs corresponding to the target genes (PLA2G4E, FABP4, and OXSM), which is significantly enriched in BP, MF, and KEGG. These are also the IncRNAs that need more attention in further experiments. 
The interaction between 18 DELs and lipid metabolism-related target genes is shown in Fig. 8. A IncRNA may interact with multiple lipid metabolism-related genes, and a gene may also be regulated by multiple IncRNAs. Among the target genes related to lipid metabolism, the expression trend of significantly upregulated or downregulated genes is consistent with the targeted regulation of DELs, which is not only in line with the general mode of action of IncRNAs on genes but also the focus of subsequent experiments.

\section{Construction of IncRNA_miRNA_mRNA interaction regulation network}

The differential miRNAs used in this section were derived from the small RNA sequencing results of the research team. LncRNAs can act as precursor molecules of miRNAs. When looking for IncRNAs that interact with miRNAs, it is necessary to filter out IncRNAs that may be precursors of miRNAs. Based on the homology between IncRNAs and miRNAs precursors, we use Blastn software to search for such IncRNAs, and the results are listed in Supplementary Table 7. Then, the IncRNAs corresponding to differential miRNAs were predicted by miRanda software, and miRNAs regulated by the DELs are screened. The target genes of differential miRNAs were predicted and cross-differentiated by using two software, miRanda, and RNAhybrid. Based on ceRNA theory, We searched the IncRNAs_target gene pairs with the same miRNAs binding sites, construction of IncRNA_miRNA_mRNA regulatory relationship with IncRNAs as a decoy, miRNAs as core and mRNAs as the target, and calculation of Pearson correlation coefficients for the expression of IncRNA_miRNA and miRNA_mRNA (Fig. 9).

As shown in the regulatory network diagram, all lipid metabolism-related target genes SYK and PPAP2C (cis) are also the target genes of lipid metabolism DELs, constituting the regulatory relationships of TCONS_00054231_bta-miR-455-3p_SYK, TCONS_00184475_bta-miR-455-3p_SYK, and TCONS_00027906_bta-miR-331-3p_PPAP2C. The upregulated genes DIS3L2, SRRM2, NDRG1 and the downregulated genes $A N O 1$ and MTM1 are the DEGs screened in this study, and 18 ceRNAs regulatory networks contain these DEGs. Also, lipid metabolism-related DELs are involved in a total of 76 ceRNAs regulatory networks, including 10 IncRNAs, 8 miRNAs, and 32 genes. There are 80 ceRNAs regulatory networks involved in lipid metabolism-unrelated DELs, consisting of 8 IncRNAs, 6 miRNAs, and 24 genes, are enriched for target genes GO/KEGG as shown in Supplementary Table 8. Downregulated bta-miR2387 is a differential miRNAs with the largest number of potential lipid metabolism-related target genes (13) and only interacts with lipid metabolism-related differential IncRNA TCONS_00172817. Non-lipid metabolism-related differences IncRNA TCONS_00004679, TCONS_00091027, and TCONS_00083670 may all play a regulatory role in bta-miR-2387.

It has been shown that the downregulation of bta-miR-145 among many differential miRNAs can regulate the lipid droplet formation and TAG accumulation [15]. Therefore, the regulatory relationship centered on bta-miR-145 is one of the focuses of our subsequent experiments. Notably, TCONS_00027906, TCONS_00054231, TCONS_00082721, TCONS_00045874, and TCONS_00083670 act together on bta- 
miR-145, which in turn regulates the expression of $F A S N$, which was a potential regulatory network for probing the mechanism of milk fat regulation of the potential regulatory network. The downregulated expression of bta-miR-421, bta-miR-331-3p and bta-miR-197 was a lipid metabolism DELs that specifically targets lipid metabolism-related differential miRNAs, while bta-miR-222 was a non-lipid metabolism differential IncRNA specifically targets lipid metabolism-related differential miRNAs. Interestingly, among the numerous lipid metabolism-related differential miRNAs, only bta-miR-370 is an upregulated differential miRNA, while the rest are downregulated.

Within the entire regulatory network relationship, the differential miRNAs were negatively correlated with DELs expression in all samples (8) with a correlation coefficient of -0.10 to -0.75 . There was a negative correlation between differential miRNAs and the expression of some target genes (- 0.10 to -0.67$)$. e.g.: bta-miR-370 with MTM1, ANO1, PLA2G15, FADS2 genes; bta-miR-2387 with SRRM2, NDRG1, PPP2R5A genes; bta-miR-455-3p with SMAD2, HADHB genes; bta-miR-1343-3p with RXRA, DIS3L2, PLA2G4F genes; bta-miR-331-3p with PLTP, SPHK2, LEP, CYP26B1, TBL1X genes; bta-miR-197 with TBL1X genes (Supplementary Table 9). We need to focus on the IncRNA_miRNA_mRNA interaction regulatory network with negative correlation and targeted relationship in the subsequent experiments.

\section{Protein interaction network analysis}

Protein interaction network analysis of DEGs (11) screened the possible regulation of milk lipid metabolism, target genes predicted by the co-localization and co-expression of DELs (17), and lipid metabolism-related genes corresponding to differential miRNAs targeted by DELs (34), the results are shown in Fig 10. There are 49 nodes and 113 interaction link sets in the protein interaction network diagram, with an enriched $P$-value of 1.0e-16 and a confidence level of 0.4 (moderate). The different colored bonds represent the source of evidence for protein interactions, and the more bonds involved in biological functions, the more likely they are. The PLTP $P P P 2 R 5 A \square S O C S 5 \square A P O C 3 \square V A R S 2 \square / T G A 6 \square P D G F D \square$ BCAT1 $\square A T P 8 A 2 \square K C N M A 1 \square Z F Y V E 28 \square C E S 4 A$ and $C T S H$ genes are not represented in the figure because

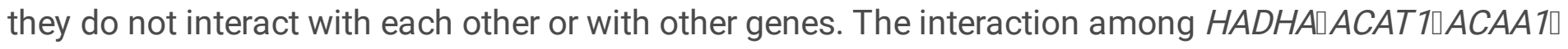
HADHB\ACADS and ACADSB genes, FABP3, FABP4, and FABP7 genes, FASN, OXSM, and MCAT genes are strongly supported by the data with very high intensity as can be seen from the figure. Secondly, $S Y K$ and PLCG1, TNF and JUN, PPAP2C and SPHK2, TBL $1 X$ and RXRA may also have shared functions between genes. Notably, MAPK3 genes has a strong interaction with several lipid metabolism-related genes (JUN, SMAD2, GH1, SPHK2, FASN, PTPRR, PLA2G4E, PLA2G4F, PLA2G4D, FGF1, and PLCG1), respectively, and may jointly play an important role in regulating milk lipid metabolism. The remaining genes also interacted with each other. Although the data support is relatively small, they still have some reference value.

\section{The expression levels of DELs and DEGs were verified by qRT-PCR}


We randomly selected 8 DELs and 8 DEGs, analyzed their relative expression levels by qRT-PCR in high and low groups (three repeats), and converted the difference multiplea by $\log _{2}$ FoldChange. As shown in Fig. 11, the results of the qRT-PCR were consistent with those of RNA-seq, which confirmed the accuracy of transcriptome sequencing.

\section{Discussion}

\section{Candidate DEGs and DELs in milk fat metabolism}

It has been confirmed that many genes involve in the lactation initiation, maintenance and growth and development of the mammary gland through direct or indirect regulation [16-18]. In this study, a total of 11 candidate DEGs related to lipid metabolism were screened and enriched in PI3K-Akt, MAPK, and Wnt lipid metabolism-related pathways $[19,20]$. The downregulated ENPP2 gene is significantly enriched in BP, MF, and lipid metabolism-related pathways, indicating that it has a greater possibility of regulating milk fat metabolism. Moreover, studies have shown that the lack of ENPP2 has a significant protective effect on hepatic steatosis [21]. Dong et al. [22] found that the expression of PDGFD in adipose tissue of obese individuals was higher than that of lean individuals. In this study, PDGFD was also highly expressed in BMECs with high MFP $\triangle$ meanwhile, the downregulated gene KCNMA1 also showed a downregulated trend in white adipose tissue and hypertrophied adipocytes of mice on the high-fat diet [23], which confirmed the reliability of sequencing, suggesting that PDGFD and KCNMA1 genes may play an important role in milk fat metabolism. BCAT1 is a branched-chain aminotransferase that has been shown to mainly affect MFP, milk protein percentage, and milk yield of dairy cows [24]. CES4A has also been shown to be mainly involved in lipid metabolism [25]. However, how BCAT1 and CES4A regulate milk fat synthesis has not yet been reported This will be the focus of our future research. It is worth noting that the latest literature reportes that the differentially downregulated $D K K 1$ related to lipid metabolism seems to play a significant role in promoting obesity in mice [26], which is inconsistent with the results of this study. It may be related to different species or tissues in the study. Other lipid metabolism-related DEGs APOL 1, ATP8A2, PTPRR, ZFYVE28, and CTSH have been reported to associate with kidney disease [27], nerve disease [28], ovarian cancer[29], glomerular filtration barrier [30] and atherosclerosis [31].

LncRNAs can regulate gene expression within $100 \mathrm{~kb}$ (cis), and can also change the expression of distant mRNAs (trans) [32]. The functional enrichment analysis of 38 target genes of DELs showed that 17 target genes (10 co-expression and 7 co-localization) were enriched in lipid metabolism-related pathways. Among them, $F A B P 3, F A B P 4$, and $F A B P 7$ are trans-target genes. Studies have confirmed that these genes encode highly abundant fatty acid-binding proteins in bovine mammary glands and transport long-chain fatty acids to the endoplasmic reticulum to synthesize TAG [33]. Protein interaction network analysis showed that the interactions between $F A B P 3, F A B P 4$, and $F A B P 7$ were also strongly supported by data, which further indicated that they may have similar functions and participate in common BP.

Based on bioinformatics analysis, a total of 18 candidate DELs that may affect milk fat synthesis in dairy cows were screened out. A IncRNA may target multiple genes related to lipid metabolism, and a gene may 
be regulated by multiple IncRNAs [11]. These results indicated that IncRNAs were diverse in regulating the function of target genes. In addition, some of the lipid metabolism-related target genes are also significantly upregulated or downregulated. The target genes expression trends are consistent with the DELs that may be targeted and regulated, which is consistent with the general mode of action of IncRNAs on genes [34], and further reflects the accuracy of the sequencing results. It is worth noting that FABP4 is a gene encoding a highly rich fatty acid-binding proteins in the mammary gland, which may have a targeting relationship with TCONS_00082721 and TCONS_00172817 [22]. Among all IncRNAs related to lipid metabolism, TCONS_00082721, TCONS_00119434, TCONS_00191498, TCONS_00007612, TCONS_00118412, and TCONS_00163391 are long intervening/intergenic noncoding RNAs (lincRNAs). Because lincRNAs do not overlap with exon sequences in organisms and show a higher degree of tissue specificity [35] and disease specificity [36]. They are involved in chromatin modification, epigenetic regulation, genomic imprinting, transcriptional control, and mRNA processing before and after translation [37]. Therefore, lincRNAs related to milk fat metabolism will be the focus of our subsequent studies. The IncRNAs that might regulate milk fat metabolism are predicted by co-localization and co-expression to be TCONS_00104103, TCONS_00162586, TCONS_00172817, TCONS_00082721, and TCONS_00143117. Interestingly, the lincRNA TCONS_00082721 with FABP4 as the candidate target gene is also a DELs that regulates milk fat metabolism predicted by the co-localization and co-expression. To clarify this hypothesis, it needs to be further verified in functional experiments. These findings will be of great significance for further research on the mechanism of milk fat synthesis in dairy cows.

\section{Protein interaction and LncRNA_miRNA_mRNA regulatory network analysis}

As an important regulatory factor, miRNAs can change the expression of target mRNA and involve widely in various biological processes. In recent years, more and more studies have shown that IncRNAs have the function of sponge adsorption of miRNAs and can shield the inhibition or degradation of target genes by competitive binding of miRNAs. And rapidly upregulate its expression [38, 39]. In this study, we first predicted the targeted differential miRNAs of DELs and then predicted the target genes of differential miRNAs. Thirty-four target genes related to lipid metabolism of differential miRNAs with DELs targeting relationship were screened out by GO/KEGG enrichment analysis.

Protein interaction network analysis showed that the target genes HADHA, HADHB, ACAA1, ACADS, $A C A D S B$, and $A C A T 1$ had a strong interaction relationship and might have common functions. HADHB is involved in the $\beta$-oxidation of fatty acids and catalyzes the second to fourth steps of the $\beta$-oxidation of fatty acid. The other five genes are acyltransferases or dehydrogenases, which play important regulatory roles in the $\beta$-oxidation of fatty acids [40]. Also, the interaction between FASN, OXSM, and MCAT genes is strongly supported by the data. FASN is an enzyme involved in fatty acid (FA) synthesis and plays a significant role in the formation of new adipogenesis in mammals. It has been found that the variation of FASN is related to a variety of dairy traits, including milk fat content, total milk solid content and peak milk yield, etc [41]هShi et al. [42] found that $F A S N$ variation can be used as a genetic marker to improve 
milk fat content and milk solid level of yaks. The co-overexpression of SCAP+SREBP1 will also lead to the increase in the abundance of FASN gene mRNA and the formation of lipid droplets [43]. In short, the current study on the FASN gene in the milk fat synthesis has been relatively mature at present. OXSM is a 3-oxyacyl -[acyl carrier protein] synthase. Xiong et al. [44] found that OXSM has inhibitory effects on fat catabolism, fat anabolism, and fatty acid oxidation, and promotes glycerol transport and polyunsaturated fatty acid synthesis. MCAT is also an important gene involved in lipid metabolism [45]. Therefore, there is an inevitable interaction between FASN, OXSM, and MCAT genes, and they all play a potential role together in the regulation of lipid metabolism. MAPK3 plays a major role in autophagy, and there is evidence that MAPK3 is involved in lipid metabolism [46]. We also found that the MAPK3 gene has a strong interaction with multiple lipid metabolism-related genes (JUN, SMAD2, GH1, SPHK2, FASN, PTPRR, PLA2G4E, PLA2G4F, PLA2G4D, FGF1, and PLCG1). It is speculated that this gene may play a crucial role in the lipid metabolism process, and the detailed mechanism needs further study.

Through functional analysis, a total of 156 milk fat metabolism-related ceRNAs were constructed in this study. Among the miRNAs targeted by DELs, miR-145 has been shown to regulate lipid drop-formation and TAG accumulation in goat mammary epithelial cells [15]. The regulatory network centered on miR145 will be the focus of subsequent experimental research. Since TCONS_00082721 is a differential lincRNA predicted by co-localization and co-expression that may regulate milk fat metabolism, the competitive regulatory relationship between TCONS_00082721_miR-145_FASN and TCONS_00082721_miR-145_TNF will be further studied and cell experiments will be carried out to verify the interaction and function between them. The target gene predicted by TCONS_00172817 is FABP4 [22], which encodes a highly abundant fatty acid-binding protein in the mammary glands of dairy cows. These IncRNAs may regulate milk fat metabolism predicted by the co-localization and co-expression. Therefore, the ceRNAs regulatory network related to TCONS_00172817 is likely to be involved in the process of milk fat synthesis and needs attention. For non-lipid metabolism DELs,TCONS_00045874 and TCONS_00083670 may play a role in miR-145, regulating the expression of lipid metabolism-related genes FASN [43] and TNF [47]. Among all the DELs related to lipid metabolism targeted differential miRNAs, bta-miR-2387 has the most abundant predicted target genes, which may be an important gene in the process of milk fat synthesis in dairy cows. Therefore, the ceRNAs regulatory network with bta-miR2387 as the core will have more choices in functional verification of its interaction and function, and the possibility of success will also be greater. To sum up, the ceRNAs network analysis was performed on the transcriptome data (mRNA, miRNAs, IncRNAs) obtained from BMECs with high and low MFP. It provides a new way to understand the function of genes in the biological process of milk fat metabolism, and it also provides a new perspective for studying the lactation process of dairy cows.

\section{Conclusion}

In this study, we isolated BMECs of Holstein cows with extreme differences MFP from fresh milk and measured IncRNAs and mRNA transcriptome profiles. Through functional enrichment analysis, 11 DEGs and 18 DELs related to milk fat metabolism were screened out, and 156 IncRNA_miRNA_mRNA interaction regulation networks related to milk fat metabolism were constructed. The results of this study 
provide a new way to understand the gene function during milk fat synthesis and provide many new insights into the structure of molecular pathways during lactation.

\section{Materials And Methods \\ Ethics statement}

In this study, BMECs were isolated and cultured from milk samples of dairy cows. $200 \mathrm{~mL}$ milk samples were collected from each dairy cow, and aseptic operation was strictly carried out during the sampling process, so there was no harm to dairy cows.

\section{Experimental cow screening and milk sample collection}

245 Holstein cows were selected from Ningxia Nongkeng Heilanshan Maosheng dairy farm with the same breeding background, similar lactation (150-220 days) and age. Early, middle and late milk samples of each cow were collected for dairy herd improvement (DHI) determination. Holstein cows with high MFP $(n=4)$ and low MFP $(n=4)$ with somatic cell count (SCC) less than $100000 / \mathrm{mL}$ were selected (Table 3). Fresh milk samples were aseptically collected into $50 \mathrm{~mL}$ centrifuge tubes, and four tubes per cow were collected for a total of $200 \mathrm{~mL}$. Tighten the cap of the bottle, seal and sterilize it, put it in a thermos flask containing $37^{\circ} \mathrm{C}$ sterile water, and return it to the laboratory for later use.

\section{Isolation and identification of primary BMECs}

The composition of the complete culture medium and culture method were the same as those of Duan et al. [48]. When the cells grew to $80 \%$ ( $F_{2}$ generation), total cellular RNA (TaKaRa; D9108A) was extracted. To exclude genomic DNA contamination, DNAase (TaKaRa; 00079459) was used for reverse transcriptional preprocessing (TaKaRa; BK3702). Primers were designed using Primer Premier 5.0 and PCR amplification was performed for genes specifically expressed in BMECs related to milk fat synthesis and keratin 8 (TaKaRa; DR100A).

PCR reaction procedure: pre-denaturation at $94^{\circ} \mathrm{C}$ for $4 \mathrm{~min}$, denaturation at $94^{\circ} \mathrm{C}$ for $30 \mathrm{~s}$, annealing (Supplementary Table 10 for annealing temperature) for $30 \mathrm{~s}$, extension at $72{ }^{\circ} \mathrm{C}$ for $30 \mathrm{~s}, 35$ cycles, $72{ }^{\circ} \mathrm{C}$ extensions for $10 \mathrm{~min}$.

The basic identification of BMECs is the same as that of Shen et al. [14]. The triglyceride (TAG) content of BMECs was determined by using the triglyceride enzymatic assay kit (E1025) of the high-fat samples. Then the protein concentration of the sample was measured by using the BCA protein content detection kit, and the TAG content was adjusted for the protein concentration per mg.

\section{Library construction and transcriptome sequencing}


The Trizo method was used to extract total RNA from BMECs (F2) of high and low MFP cows (4 heads), and the quality of RNA samples was strictly controlled. The integrity of RNA and DNA contamination were analyzed by agarose gel electrophoresis. The concentration and purity of RNA (OD260/280) were detected by Nanodrop, and the integrity of RNA was accurately detected by Agilent 2100 bioanalyzer. The $260 / 280$ ratio of all samples was approximately 2.0 , and the RNA integrity index (RIN) was $\geq 8.0$. After determining the RNA purity and quality, ribosomal RNA was removed to construct strand-specific library.

After passing the library inspection, Illumina PE150 sequencing was performed. After the original data is obtained, the reads with adapter, $\mathrm{N}$ (undetermined base information) ratio greater than 0.002 , and lowquality bases with a read length of more than $50 \%$ are removed. After sequencing error rates (Q20 and Q30) and GC content distribution checks, clean reads for subsequent analysis were obtained. tophat2 (http://tophat.cbcb.umd.edu), Hisat2 (http://ccb.jhu.edu/software/hisat2), and STAR (http://code.google.com/p/rna-star) software were used to compare and analyze the RNA sequencing(RNA-seq) data.

\section{DEGs, DELs screening, and functional enrichment analysis}

The level of gene expression was quantified using fragments per kilobase of exon per millions of reads (FPKM) value. Expression difference significance analysis was performed using edgeR software. The corrected $P$-value (Padj) was used to determine the significance level, and Padj $<0.05$ was used as the standard of differential significance, while |log2FoldChange|>1.5 was another important criterion for screening DELs and DEGs. Co-localization of IncRNAs with protein-coding genes was used to find genes within $100 \mathrm{~kb}$ upstream and downstream of IncRNAs, and co-expression was used to predict the target genes of IncRNAs. The gene ontology (GO) database was used to enrich cellular composition (CC), biological process (BP), and molecular function (MF) of DEGs and target genes of DELs. Kyoto encyclopedia of genes and genomes (KEGG) was used to identify the major biochemical metabolic pathways and signal transduction pathways involved in genes. The protein interaction network between DEGs and DELs target genes was analyzed by STRING database (https://string-db.org/) and further visualized using Cytoscape (http://www.cytos-cape.org/).

\section{MiRNA target prediction}

miRanda database was used with the default parameters to identify conserved miRNA target sites in the 3'UTR of IncRNAs. MiRNAs target gene prediction was based on the intersection of miRanda (Total score $\geq 140$, Total energy $\leq-15 \mathrm{Kcal} / \mathrm{mol}$ ) and RNAhybrid (mfe $\leq-20 \mathrm{Kcal} / \mathrm{mol}, P<0.05)$.

\section{qRT-PCR validation of sequencing data}


To confirm the sequencing results, eight DEGs and DELs were randomly selected for qRT-PCR validation, respectively. First-strand cDNA synthesis was performed using the PrimeScript RT kit (Takara, Dalian, China). qRT-PCR (three replicates) was performed by SYBR Premix Ex Taq ${ }^{\mathrm{TM} I I}$ (TaKaRa) on the Bio-Rad CFX96 Touch $^{\text {TM }}$ Real-Time PCR Detection System (Bio-Rad, Hercules, CA, USA). Amplification procedure: $95^{\circ} \mathrm{C}$ for $30 \mathrm{~s}, 95^{\circ} \mathrm{C}$ for $5 \mathrm{~s}$, annealing for $30 \mathrm{~s}, 40$ cycles. qRT-PCR primers were designed using Primer Premier 5.0 with primers spanning at least one intron, and the sequence and annealing temperature of each primer are shown in Supplementary Table 10.

\section{Data analysis}

The data were filtered by Microsoft Excel 2016, and the relative expressions of DEGs and DELs were analyzed by the $2^{-\triangle \triangle C t}$ method and normalized by the GAPDH gene. The data were analyzed using SAS 9.2 (SAS Institute, Cary, NC, USA) with a linear mixed model for one-way ANOVA.

\section{Abbreviations}

LncRNAs: long noncoding RNAs; RNA-seq: RNA sequencing; BMECs: bovine mammary epithelial cells; MFP: milk fat percentage; DEGs: differentially expressed genes; DELs: ifferentially expressed IncRNAs; lincRNA: long intervening/intergenic noncoding RNA; miRNAs: microRNAs; qRT-PCR: quantitative real-time reverse transcription PCR; ncRNAs: non-coding RNAs; DHI: dairy herd improvement; SCC: somatic cell count; TAG: triglyceride; FPKM: quantified using fragments per kilobase of exon per millions of reads; Padj: the corrected $P$-value; GO: gene ontology; CC: cellular composition; BP: biological process; MF: molecular function; KEGG: kyoto encyclopedia of genes and genomes.

\section{Declarations}

\section{Ethics approval and consent to participate}

The milk sample from live animals were studied in this study. The Animal Ethics Committees of Ningxia University approved the experimental design and animal sample collection for the present study (permit number NXUC20200619). And animal experiments were conducted strictly followed the guidelines of the Regulations for the Administration of Affairs Concerning Experimental Animals (Ministry of Science and Technology, China, 2004).

\section{Consent for publication}

Not applicable.

\section{Availability of data and materials statement}


All data generated or analyzed in this study are included in this article [and its supplementary information file], and the datasets have been submitted to the SRA database with the accession number PRJNA730595. Access to the data of permanent link to https://www.ncbi.nlm.nih.gov/sra/PRJNA730595.

\section{Competing interests}

The authors declare that we have no competing interests.

\section{Financial support statement}

This project is supported by the special breeding project of high-quality and high yield dairy cows in the Ningxia Autonomous region (Grant No: 2019NYYZ05).

\section{Author contributions}

TM: Sample collection, data processing and article writing. HHH: Sample collection, article modification and visualization. XFF, YW, BJY, JML, WW and JZ: Sample collection and verified by qRT-PCR. YFM: Article grammar modification. YLG: Conceptual analysis, writing-review and editing.

\section{Acknowledgments}

Thanks to all the teachers who helped with this experiment, and to all the authors of this paper for their hard work.

\section{References}

1. Li D, Xie X, Jie W, Bian Y, Li Q, Gao X, Wang C, Dettman RW. MiR-486 regulates lactation and targets the PTEN gene in cow mammary glands. Plos One. 2015; 10(3): e118284.

2. Chen Z, Chu S, Wang X, Fan Y, Zhan T, Arbab A, Li M, Zhang H, Mao Y, Loor JJ. MicroRNA-106b regulates milk fat metabolism via ATP binding cassette subfamily A member 1 (ABCA1) in bovine mammary epithelial cells. J AGR FOOD CHEM. 2019; 67(14): 3981-3990.

3. Bauman DE, Mather IH, Wall RJ, Lock AL. Major advances associated with the biosynthesis of milk. J DAIRY SCl. 2006; 89(4): 1235-1243.

4. Xu B, Isabelle G, Miao H, Dang VP, Johnson CN, Xu R, Chen XW, Cawthorn WP, Macdougald OA, Koenig RJ. Multiple roles for the non-Coding RNA SRA in regulation of adipogenesis and insulin sensitivity. Plos One. 2010; 5(12): e14199. 
5. Ma Q, Li L, Tang Y, Fu Q, Liu S, Hu S, Qiao J, Chen C, Ni W. Analyses of long non-coding RNAs and mRNA profiling through RNA sequencing of MDBK cells at different stages of bovine viral diarrhea virus infection. Res Vet Sci. 2017; 115: 508-516.

6. Xu J, Feng L, Han Z, Li Y, Wu A, Shao T, Ding N, Li L, Deng W, Di X. Extensive ceRNA-ceRNA interaction networks mediated by miRNAs regulate development in multiple rhesus tissues. Nucleic Acids Res. 2016; 44(19): 9438-9451.

7. Fatica A, Bozzoni I. Long non-coding RNAs: new players in cell differentiation and development. Nat Rev Genet. 2014; 15(1): 7-21.

8. Yeong-Hwan L, Duk-Hwa K, Jaetaek K, Jin PW, Hyun K, Young-Kook K, Seungil R. Identification of long noncoding RNAs involved in muscle differentiation. Plos One. 2018; 13(3): e193898.

9. Yu S, Zhao Y, Lai F, Chu M, Hao Y, Feng Y, Zhang H, Liu J, Cheng M, Li L. LncRNA as ceRNAs may be involved in lactation process. Oncotarget. 2017; 8(58): 98014-98028.

10. Chao T, Chen Q, Zhao L, Ma J, Ibeagha-Awemu EM. Identification and characterization of long intergenic noncoding RNAs in bovine mammary glands. BMC Genomics. 2017; 18(1): 468.

11. Zheng $X$, Ning C, Zhao P, Feng W, Jin Y, Zhou L, Yu Y, Liu J. Integrated analysis of long noncoding RNA and mRNA expression profiles reveals the potential role of long noncoding RNA in different bovine lactation stages. J Dairy Sci. 2018; 101(12): 11061-11073.

12. Lu C, Yang R, Liu B, Li Z, Zhao Z. Establishment of two types of mammary epithelial cell lines from chinese Holstein dairy cow. J Anim Vet Adv. 2012; 11(8): 1166-1172.

13. Hu H, Wang J, Bu D, Wei H, Zhou L, Li F, Loor JJ, Aziz SA. In vitro culture and characterization of a mammary epithelial cell Line from chinese Holstein dairy cow. Plos One. 2009; 4(11): e7636.

14. Shen B, Zhang L, Lian C, Lu C, Zhang Y, Pan Q, Yang R, Zhao Z. Deep sequencing and screening of differentially expressed microRNAs related to milk fat metabolism in bovine primary mammary epithelial cells. Int J Mol Sci. 2016; 17(2): 200.

15. Wang H, Shi H, Luo J, Yi Y, Yao D, Zhang X, Ma G, Loor JJ. MiR-145 regulates lipogenesis in goat mammary cells via targeting INSIG1 and epigenetic regulation of lipid-related genes. J Cell Physiol. 2017; 232(5): 1030-1040

16. Dai WT, Zou YX, White RR, Liu JX, Liu HY. Transcriptomic profiles of the bovine mammary gland during lactation and the dry period. Funct Integr Genomic. 2017; 18(2): 125-140.

17. Williams MM, Vaught DB, Joly MM, Hicks DJ, Sanchez V, Owens P, Rahman B, Elion DL, Balko JM, Cook RS. ErbB3 drives mammary epithelial survival and differentiation during pregnancy and lactation. Breast Cancer Res. 2017; 19(1): 105.

18. Bach K, Pensa S, Grzelak M, Hadfield J, Adams DJ, Marioni JC, Khaled WT. Differentiation dynamics of mammary epithelial cells revealed by single-cell RNAsequencing. Nat Commun. 2017; 8(1): 2128.

19. Bing Y, Jiao B, Ge W, Zhang X, Wang S, Zhao H, Wang X. Transcriptome sequencing to detect the potential role of long non-coding RNAs in bovine mammary gland during the dry and lactation period. BMC Genomics. 2018; 19(1): 605. 
20. Kessenbrock K, Smith P, Steenbeek SC, Pervolarakis N, Kumar R, Minami Y, Goga A, Hinck L, Werb Z. Diverse regulation of mammary epithelial growth and branching morphogenesis through noncanonical Wnt signaling. P Natl Acad Sci Usa. 2017; 114(12): 3121.

21. Brandon JA, Kraemer M, Vandra J, Halder S, Ubele M, Morris AJ, Smyth SS, Souza-Mello V. Adiposederived autotaxin regulates inflammation and steatosis associated with diet-induced obesity. Plos One. 2019; 14(2): e0208099.

22. Dong K, Yang M, Han J, Ma Q, Jiang L. Genomic analysis of worldwide sheep breeds reveals PDGFD as a major target of fat-tail selection in sheep. BMC Genomics. 2020; 21(1): 1-19.

23. Nishizuka M, Horinouchi W, Yamada E, Ochiai N, Osada S, Imagawa M. KCNMA1, a pore-forming subunit of BK channels, regulates insulin signalling in mature adipocytes. Febs Lett. 2016; 43724380 .

24. Fang M, Fu W, Dan J, Qin Z, Sun D, Ding X, Liu J, Stephen M. A Multiple-SNP approach for genomewide association study of milk production traits in Chinese Holstein cattle. Plos One. 2014; 9(8): e99544.

25. Holmes RS, Wright MW, Laulederkind S, Cox LA, Hosokawa M, Imai T, Ishibashi S, Lehner R, Miyazaki $M$, Perkins EJ. Recommended nomenclature for five mammalian carboxylesterase gene families: human, mouse, and rat genes and proteins. Mamm Genome. 2010; 21(9): 427-441.

26. Colditz J, Picke A, Hofbauer LC, Rauner M. Contributions of Dickkopf-1 to obesity-induced bone loss and marrow adiposity. JBMR Plus. 2020; 4(6): e10364.

27. Pays $E$. The function of apolipoproteins $L$ (APOLs): relevance for kidney disease, neurotransmission disorders, cancer and viral infection. FEBS J. 2021; 288(2): 360-381.

28. Choi $\mathrm{H}$, Andersen JP, Molday RS. Expression and functional characterization of missense mutations in ATP8A2 linked to severe neurological disorders. Hum Mutat. 2019; 40(12): 2353-2364.

29. Wang Y, Cao J, Liu W, Zhang J, Fan G. Protein tyrosine phosphatase receptor type R (PTPRR) antagonizes the Wnt signaling pathway in ovarian cancer by dephosphorylating and inactivating $\beta$ catenin. J Biol Chem. 2019; 294(48): 18306-18323.

30. Zambrano S, Rodriguez PQ, Guo J, Hackbarth KL, Schwarz A, Patrakka J. FYVE domain-containing protein ZFYVE28 regulates EGFR-signaling in podocytes but is not critical for the function of filtration barrier in mice. Sci Rep. 2018; 8(1): 4712.

31. Lim W, Chow V. Gene expression profiles of U937 human macrophages exposed to Chlamydophila pneumoniae and/or low density lipoprotein in five study models using differential display and realtime RT-PCR. Biochimie. 2006; 88(3-4): 367-377.

32. Guttman M, Amit I, Garber M, French C, Lin MF, Feldser D, Huarte M, Zuk O, Carey BW, Cassady JP. Chromatin signature reveals over a thousand highly conserved large non-coding RNAs in mammals. Nature. 2009; 458(7235): 223.

33. Bionaz M, Loor JJ. ACSL1, AGPAT6, FABP3, LPIN1, and SLC27A6 are the most abundant isoforms in bovine mammary tissue and their expression is affected by stage of lactation1-3. J Nutr. 2008; 138(6): 1019-1024. 
34. Cesana M, Cacchiarelli D, Legnini I, Santini T, Sthandier O, Chinappi M, Tramontano A, Bozzoni I. A long noncoding RNA controls muscle differentiation by functioning as a competing endogenous RNA. Cell. 2011; 147(2): 358-369.

35. Moran NC, Cole T, Loyal G, Magdalena K, Barbara TV, Aviv R, John LR. Integrative annotation of human large intergenic noncoding RNAs reveals global properties and specific subclasses. Gene Dev. 2011; 25(18): 1915.

36. Iyer MK, Niknafs YS, Malik R, Singhal U, Chinnaiyan AM. The landscape of long noncoding RNAs in the human transcriptome. Nat Genet. 2015; 47(3): 199-208.

37. Ulitsky I, Bartel D. lincRNAs: genomics, evolution, and mechanisms. Cell. 2013; 154(1): 26-46.

38. Wang R, Zhang S, Chen X, Li N, Li J, Jia R, Pan Y, Liang H. CircNT5E acts as a sponge of microRNA422a to promote glioblastoma tumorigenesis. Cancer Res. 2018; 78(17): 4812-4825.

39. Zhang H, Lu W. LncRNA SNHG12 regulates gastric cancer progression by acting as a molecular sponge of miR-320. Mol Med Rep. 2018; 17(2): 2743-2749.

40. Fernándezhernando C, Suárez Y, Rayner KJ , Moore KJ. MicroRNAs in lipid metabolism. Curr Opin in Lipidol. 2011; 22(2): 86.

41. Matsumoto H, Inada S, Kobayashi E, Abe T, Hasebe H, Sasazaki S, Oyama K, Mannen H. Identification of SNPs in the FASN gene and their effect on fatty acid milk composition in Holstein cattle. Livest Sci. 2012; 144(3): 281-284.

42. Shi B, Jiang $Y$, Chen $Y$, Zhao Z, Hickford J. Variation in the fatty acid synthase gene (FASN) and its association with milk traits in Gannan yaks. Animals. 2019; 9(9): 613.

43. Han LQ, Gao TY, Yang GY, Loor JJ. Overexpression of SREBF chaperone (SCAP) enhances nuclear SREBP1 translocation to upregulate fatty acid synthase ( FASN ) gene expression in bovine mammary epithelial cells. J Dairy Sci. 2018; 101(7): 6523-6531.

44. Xiong L, Pei J, Wu X, Kalwar Q, Yan P. The study of the response of fat metabolism to long-term energy stress based on serum, fatty acid and transcriptome profiles in yaks. Animals. 2020; 10(7): 1150.

45. Ghadge AA, Harsulkar AM, Diwan AG, Kuvalekar AA. Gender dependent differences in lipid metabolism in individuals with type 2 diabetes mellitus. J Diabetes Metab Disord. 2020; 19(2): 967977.

46. Xiao Y, Hao L, Yu J, Zhao Z, Guo F. MAPK1/3 regulate hepatic lipid metabolism via ATG7-dependent autophagy. Autophagy. 2016; 12(3): 592-593.

47. Alexis S, Nagadhara D, Philip N, Donna P, Ethan K, Bradley W, Tang C, Francis K, Dichek DA. ABCA1 Overexpression in Endothelial Cells In Vitro Enhances ApoAl-Mediated Cholesterol Efflux and Decreases Inflammation. Hum Gene Ther. 2018; 30(2): 236-248.

48. Duan AQ, Pang CY, Zhu P, Deng TX, Lu XR, Ma XY, Liang SS, Liang XW. Isolation, culture and Identification of buffalo mammary epithelial cells from milk. China Animal Husbandry \& Veterinary Medicine. 2017; 44(11): 3243-3249. 


\section{Tables}

Table 1

Candidate DEGs related to milk fat metabolism.

\begin{tabular}{|lllll|}
\hline Gene name & Gene location & Log2FoldChange & Up/down & Padj \\
\hline PDGFD & $15: 4259082-4536080$ & 12.0848 & Up & 0.0364 \\
\hline BCAT1 & $5: 85057073-85174596$ & 11.5674 & Up & 0.0038 \\
\hline APOL3 & $5: 74600517-74612469$ & 4.8252 & Up & 0.0089 \\
\hline ATP8A2 & $12: 33380874-33831448$ & -13.0109 & Down & 0.0008 \\
\hline PTPRR & $5: 42611538-42884729$ & -12.1701 & Down & 0.0434 \\
\hline KCNMA1 & $28: 32616314-33387186$ & -12.1505 & Down & 0.0390 \\
\hline ZFYVE28 & $6: 116265797-116364222$ & -10.5076 & Down & 0.0251 \\
\hline ENPP2 & $14: 80982673-81117148$ & -10.2255 & Down & 0.0403 \\
\hline DKK1 & $26: 6846290-6849518$ & -4.3134 & Down & 0.0061 \\
\hline CES4A & $18: 34675658-34690406$ & -2.8026 & Down & 0.0133 \\
\hline $\begin{array}{l}\text { CTSH } \\
\text { Log2FoldChange is logarithm of fold change with a base of 2, Padj is the corrected significance test } \\
\text { probability value, gene location is the specific location of the gene on the chromosome. }\end{array}$ & \\
\hline
\end{tabular}

\section{Table 2}

DELs related to lipid metabolism predicted by co-expression and co-localization. 


\begin{tabular}{|c|c|c|c|c|c|}
\hline Transcript id & LncRNA type & $\begin{array}{l}\text { Transcript } \\
\text { location }\end{array}$ & Log2FoldChange & Up/down & Padj \\
\hline TCONS_00104103 & sense_overlapping & $\begin{array}{l}\text { 23:27943191- } \\
28058062\end{array}$ & 14.3242 & Up & 0.0452 \\
\hline TCONS_00162586 & sense_overlapping & $\begin{array}{l}\text { 7:42726724- } \\
42986751\end{array}$ & 13.9441 & Up & 0.0489 \\
\hline TCONS_00172817 & antisense & $\begin{array}{l}8: 86876741- \\
87001036\end{array}$ & 13.9264 & Up & 0.0460 \\
\hline TCONS_00082721 & lincRNA & $\begin{array}{l}2: 24262990- \\
24300737\end{array}$ & 12.7310 & Up & 0.0132 \\
\hline TCONS_00143117 & antisense & $\begin{array}{l}4: 82502392- \\
82533931\end{array}$ & -12.4167 & Down & 0.0321 \\
\hline TCONS_00150126 & sense_overlapping & $\begin{array}{l}5: 28723371- \\
28948902\end{array}$ & 15.3298 & Up & 0.0005 \\
\hline TCONS_00156313 & antisense & $\begin{array}{l}6: 106460016- \\
106540196\end{array}$ & 14.2909 & Up & 0.0039 \\
\hline TCONS_00164841 & antisense & $\begin{array}{l}\text { 7:102898847- } \\
103228659\end{array}$ & 14.0298 & Up & 0.0455 \\
\hline TCONS_00119434 & lincRNA & $\begin{array}{l}27: 36076831- \\
36107350\end{array}$ & 12.7668 & Up & 0.0206 \\
\hline TCONS_00027906 & antisense & $\begin{array}{l}\text { 12:28946242- } \\
29075799\end{array}$ & 12.6328 & Up & 0.0270 \\
\hline TCONS_00054231 & antisense & $\begin{array}{l}16: 45117041- \\
45146121\end{array}$ & 12.2759 & Up & 0.0244 \\
\hline TCONS_00066920 & sense_overlapping & $\begin{array}{l}\text { 18:46165575- } \\
46169765\end{array}$ & 11.5972 & Up & 0.0086 \\
\hline TCONS_00058915 & sense_overlapping & $\begin{array}{l}17: 6204238- \\
6224363\end{array}$ & 10.2082 & Up & 0.0437 \\
\hline TCONS_00191498 & lincRNA & $\begin{array}{l}X: 124180006- \\
124280661\end{array}$ & -13.9321 & Down & 0.0077 \\
\hline TCONS_00143115 & antisense & $\begin{array}{l}4: 82475604- \\
82533958\end{array}$ & -12.4900 & Down & 0.0025 \\
\hline TCONS_00007612 & lincRNA & $\begin{array}{l}1: 95512675- \\
95550502\end{array}$ & -12.1321 & Down & 0.0421 \\
\hline TCONS_00118412 & lincRNA & $\begin{array}{l}27: 16563- \\
41295\end{array}$ & -11.9714 & Down & 0.0043 \\
\hline TCONS_00163391 & lincRNA & $\begin{array}{l}7: 53750360- \\
53759144\end{array}$ & -10.6626 & Down & 0.0182 \\
\hline
\end{tabular}



co-expression that may regulate milk fat metabolism. transcript location is the specific location of the DELs on the chromosome. Padj is the corrected significance test probability value.

\section{Table 3}

High and low MFP and SCC of Holstein cattle

\begin{tabular}{|c|c|c|c|c|c|}
\hline Item & Number & Age & Lactation days & MFP (\%) & $\operatorname{SCC}(100000 / \mathrm{mL})$ \\
\hline \multirow[t]{4}{*}{ High-milk fat group } & H_2098 & 29.81 & 186 & 4.82 & 5 \\
\hline & H_2046 & 30.57 & 189 & 4.54 & 2 \\
\hline & H_2226 & 28.56 & 160 & 4.74 & 9 \\
\hline & H_2190 & 28.89 & 157 & 4.88 & 5 \\
\hline \multirow[t]{4}{*}{ Low-milk fat group } & L_2034 & 30.57 & 187 & 2.60 & 6 \\
\hline & L_2037 & 30.5 & 175 & 2.81 & 5 \\
\hline & L_2170 & 30.43 & 207 & 2.85 & 8 \\
\hline & L_2137 & 29.41 & 150 & 2.84 & 7 \\
\hline
\end{tabular}

\section{Figures}



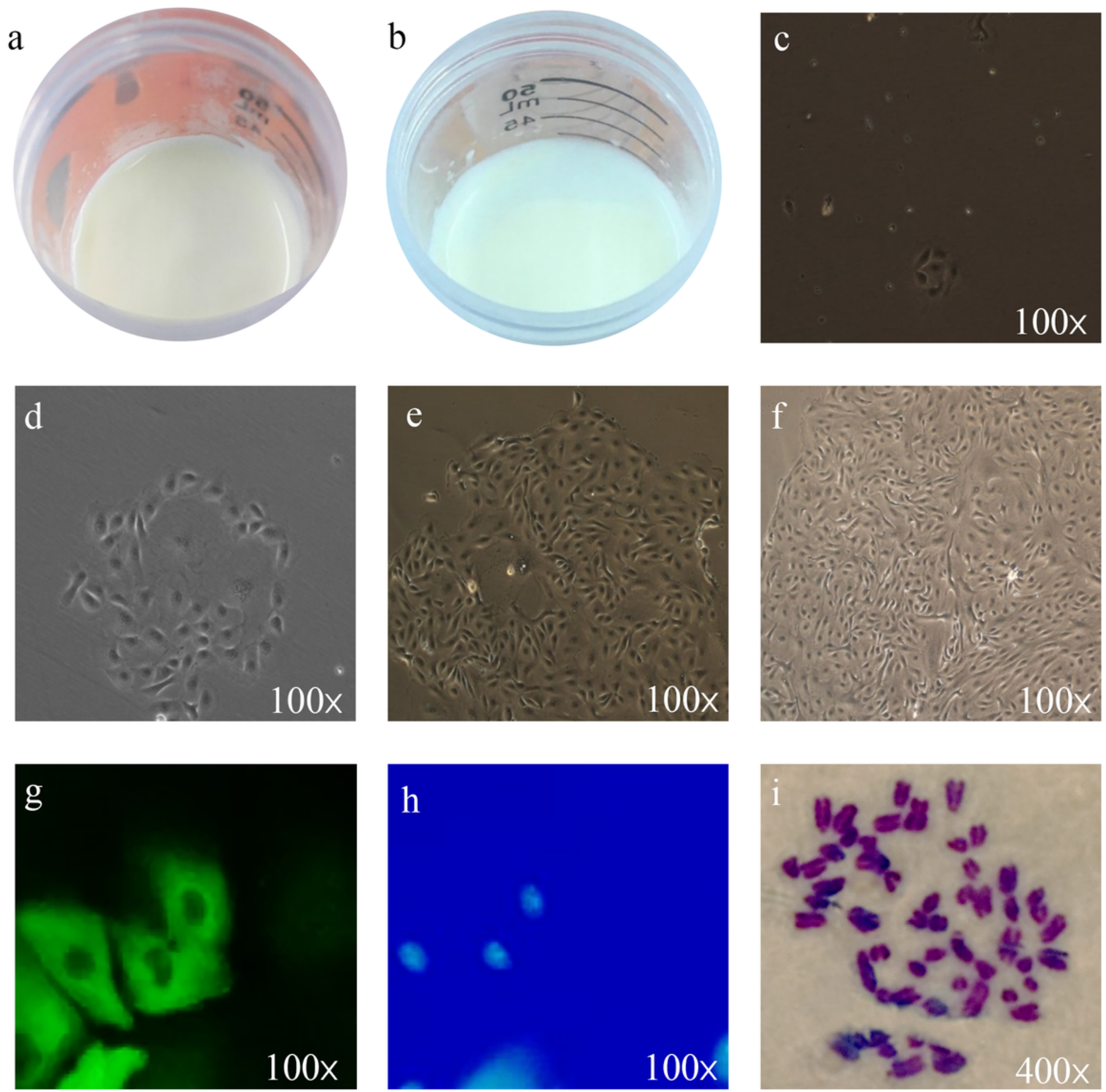

Figure 1

Isolation, culture and identification of BMECs (c-h: 10*10). a: Thickness and area of milk fat layer after centrifugation of high-milk fat milk. b: Thickness and area of milk fat layer after centrifugation of lowmilk fat milk. c: Single-cell clone of BMECs can be seen after 3 days of isolation and culture. d: Single-cell clones of BMECs gradually expand after 5 days of culture. e: BMECs have begun to proliferate rapidly after 7 days. f: After 13 days of isolation and culture, BMECs can be rapidly covered with the bottom of 
the bottle. g: BMECs labeled with green fluorescence. h: Nuclei stained blue by DAPI in the same visual field. i: Chromosomes under oil microscope.
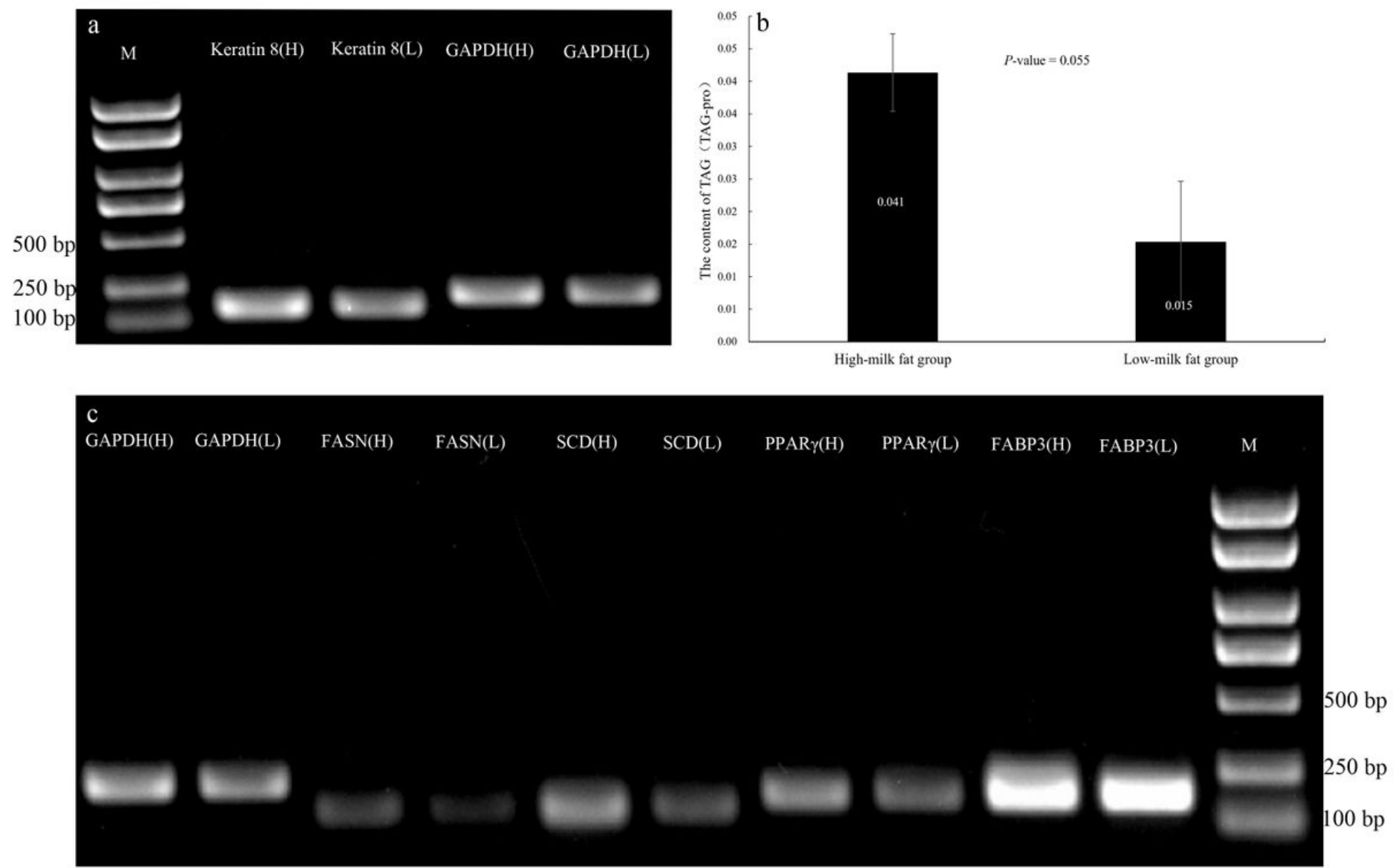

Figure 2

BMECs TAG content and expression level of adipogenic genes and keratin 8 in high and low MFP. a: Expression of keratin 8 in BMECs of dairy cows with high and low MFP. b: Content of TAG in BMECs of dairy cows with high and low MFP. c: Expression of adipogenic genes in BMECs of dairy cows with high and low MFP. M: DL2000 DNA marker, (H): high-milk fat group, (L): low-milk fat group. 
FPKM distribution

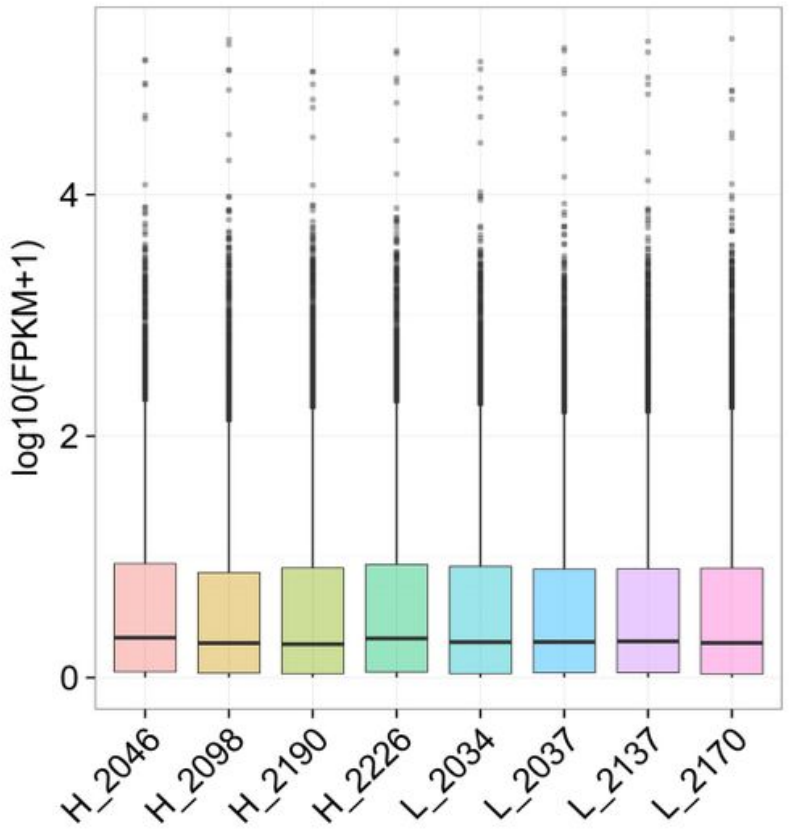

b

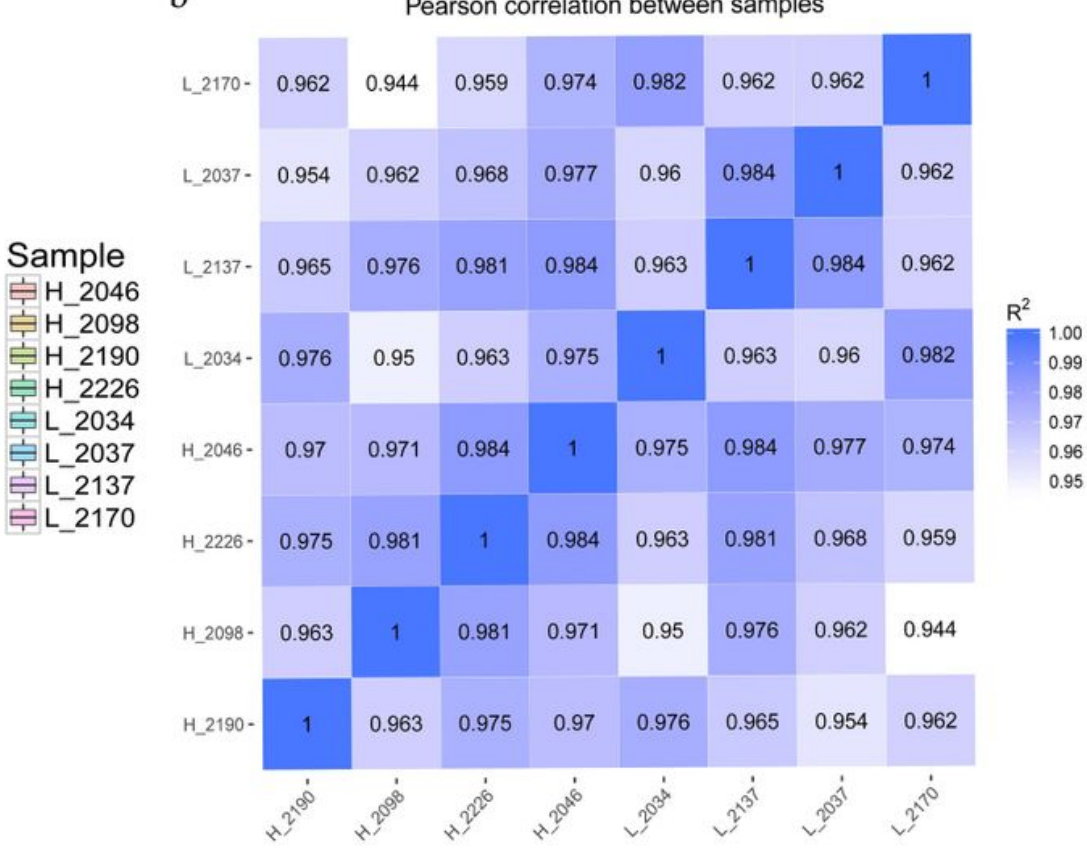

Figure 3

Expression level distribution and correlation analysis of each sample. a: The box diagram of different sample expression values, in which the abscissa is the sample name and the ordinate is $\log 10$ (FPKM+1). The box diagram of each region corresponds to five statistics (from top to bottom, the maximum, upper quartile, median, lower quartile, and minimum). b: The heat maps for the correlation analysis between samples. FPKM: fragments per kilobase of exon per million fragments mapped, R2: pearson correlation coefficient. 

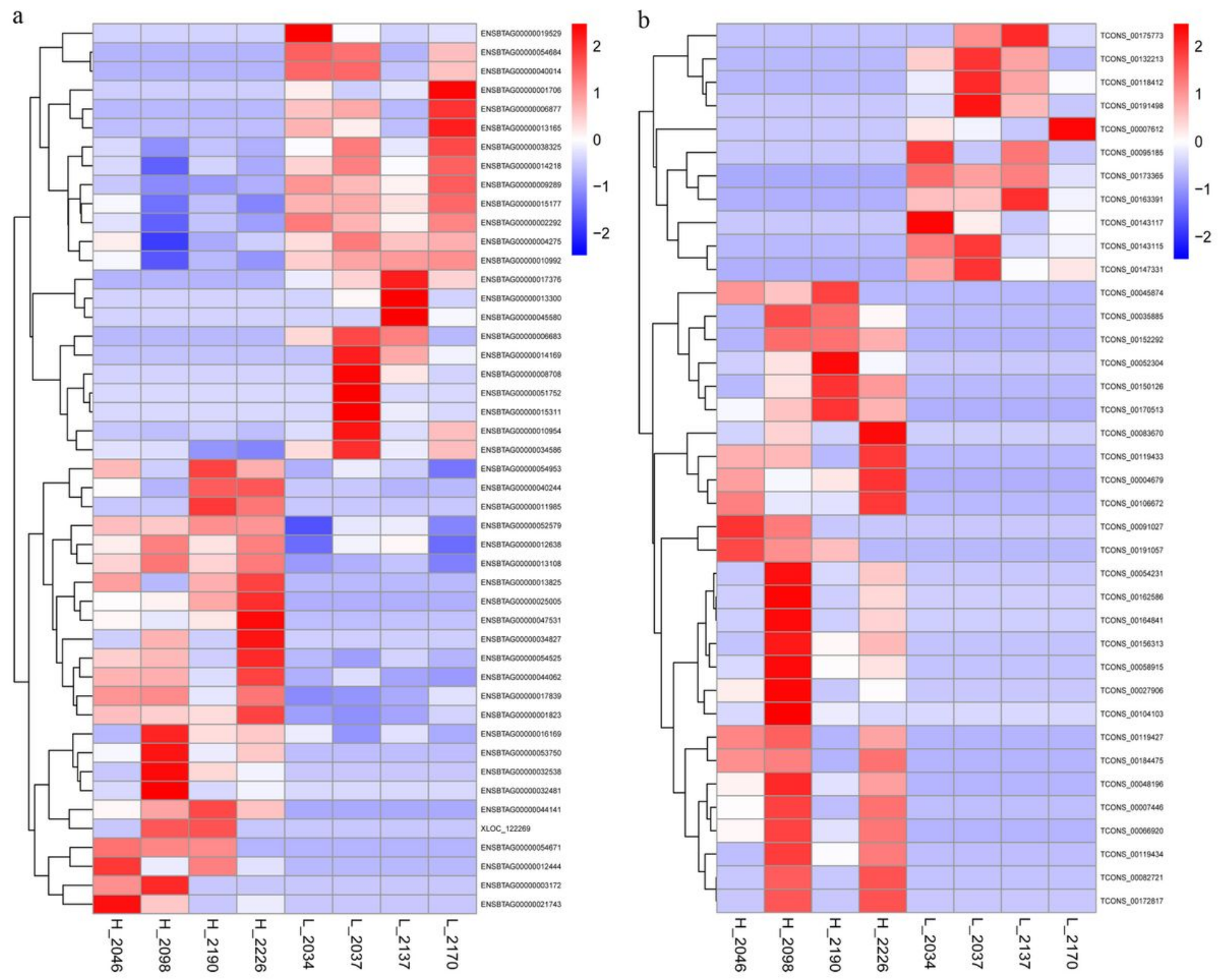

Figure 4

Hierarchical clustering of DEGs and DELs. a: The DEGs clustering heat map, the abscissa is the sample, the ordinate is the DEGs, the left side clusters the genes according to the degree of similarity of expression, the expression is gradually upregulated from blue to red, and the number is the relative expression after homogenization. b: The DELs clustering heat map. 


\begin{tabular}{|c|c|c|c|c|c|}
\hline & & & ero & genes & \\
\hline & & 4 & 9 & 13 & 17 \\
\hline $\mathrm{a}$ & & & & & \\
\hline & Negative regulation of receptor activity & & & & \\
\hline i & Regulation of angiogenesis & & & & \\
\hline ; & Regulation of vasculature development & & & & \\
\hline i & Angiogenesis & & & & \\
\hline 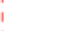 & Regulation of receptor activity & = & & & \\
\hline I & $\begin{array}{l}\text { Positive regulation of angiogenesis } \\
\text {. }\end{array}$ & & & & \\
\hline 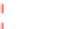 & Regulation of hormone metabolic process Negative & & & & \\
\hline $\begin{array}{l}1 \\
\vdots\end{array}$ & Regulation of mitochondrial membrane permeability & = & & & \\
\hline & Regulation of canonical Wnt receptor signaling pathway & & & & \\
\hline BP & Lung morphogenesis & a & & & \\
\hline & Negative regulation of canonical Wnt receptor signaling pathway & & & & \\
\hline 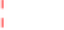 & Branched-chain amino acid biosynthetic process & = & & & \\
\hline & Leucine biosynthetic process & ] & & & \\
\hline ! & Valine biosynthetic process & [ & & & \\
\hline 1 & Negative regulation of protein homodimerization activity & a & & & \\
\hline & Neuropeptide catabolic process & - & & & \\
\hline & Blood vessel morphogenesis & & & & \\
\hline & Canonical Wnt receptor signaling pathway & & & & \\
\hline & Protein destabilization & E & & & \\
\hline & Dichotomous subdivision of terminal units involved in lung branching & D & & & \\
\hline & Extracellular region & & & & \\
\hline & Extracellular region part & & & & \\
\hline & Extracellular space & & & & \\
\hline 1 & Alveolar lamellar body & - & & & \\
\hline $\mathrm{CC}$ & Anchored to plasma membrane & $=$ & & & \\
\hline & NURF complex & = & & & \\
\hline & Mast cell granule & = & & & \\
\hline & Anchored to membrane & = & & & \\
\hline & Lamellar body & - & & & \\
\hline & ISWI-type complex & e. & & & \\
\hline & Receptor antagonist activity & 包 & & & \\
\hline & Receptor inhibitor activity & $\square$ & & & \\
\hline & HLA-A specific activating MHC class I receptor activity & 当 & & & \\
\hline & MHC class I receptor activity & 日 & & & \\
\hline & Activating MHC class I receptor activity & ] & & & \\
\hline & Aminopeptidase activity & $\theta$ & & & \\
\hline & Alkylglycerophosphoethanolamine phosphodiesterase activity & 日 & & & \\
\hline & Nucleosome-dependent ATPase activity & 日 & & & \\
\hline 1 & L-leucine transaminase activity & 是 & & & \\
\hline & L-valine transaminase activity & 日 & & & \\
\hline MF & L-isoleucine transaminase activity & 日 & & & \\
\hline & Branched-chain-amino-acid transaminase activity & 日 & & & \\
\hline & Glucokinase activity & 日 & & & \\
\hline & Fructokinase activity & 日 & & & \\
\hline & Mannokinase activity & ] & & & \\
\hline & RAGE receptor binding & ] & & & \\
\hline & Receptor regulator activity & 曰 & & & \\
\hline 1 & Hexokinase activity & 马 & & & \\
\hline & Carbohydrate binding & $\square$ & & & \\
\hline & endopeptidase activator activity involved in apoptotic process & ] & & & \\
\hline
\end{tabular}

b

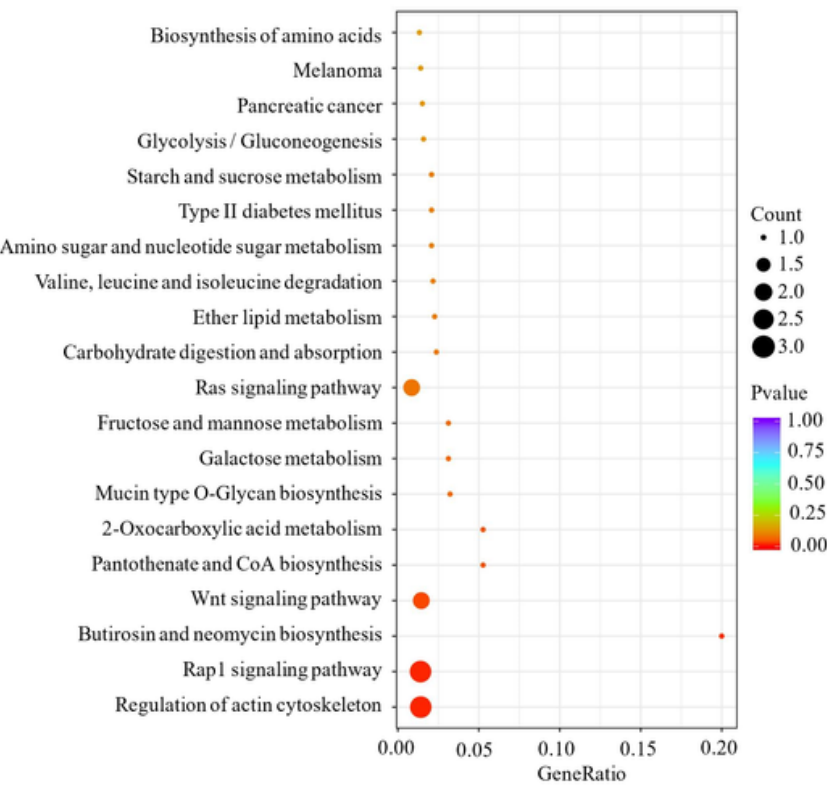

\section{Figure 5}

GO and KEGG enrichment analysis of DEGs. a: The first 20 significant $G 0$ items $(P<0.05)$, when less than 20, only show significant enrichment items, the latter is the same. b: The first 20 KEGG enrichment results. 


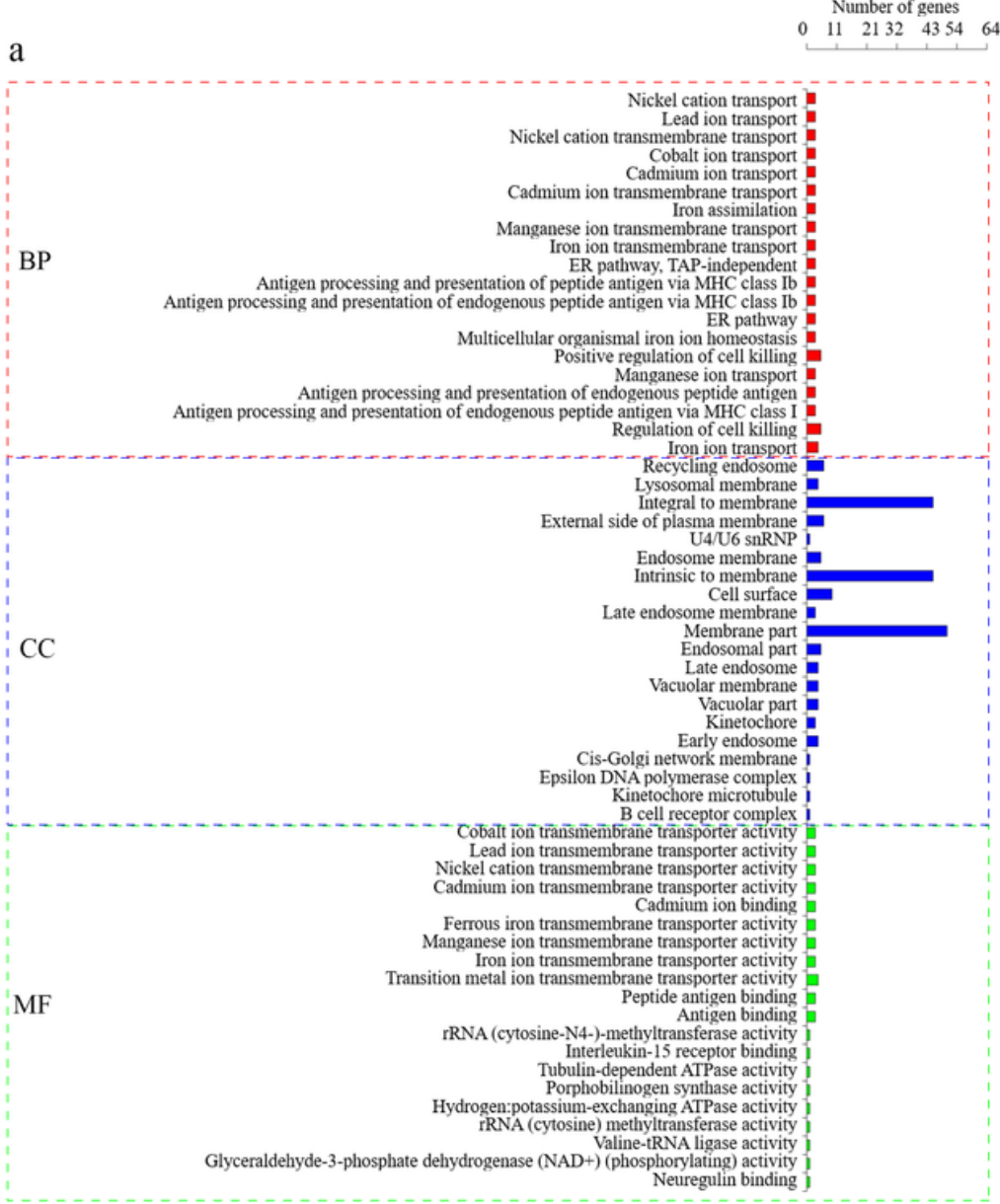

b

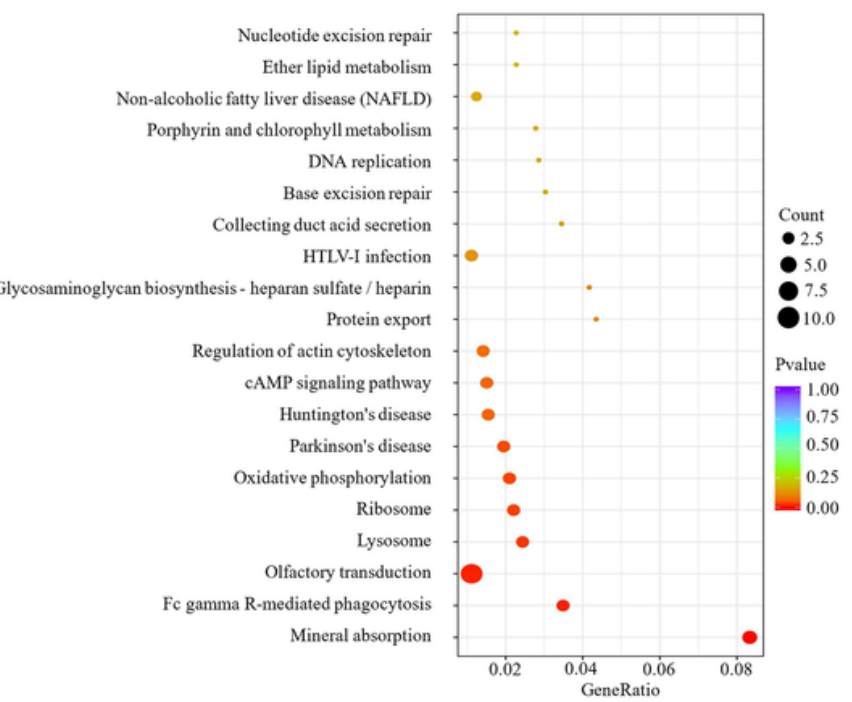

Figure 6

GO and KEGG enrichment analysis of DELs target genes predicted by co-localization. a: The first 20 significant $G O$ items $(P<0.05)$. b: The first 20 KEGG enrichment results. 


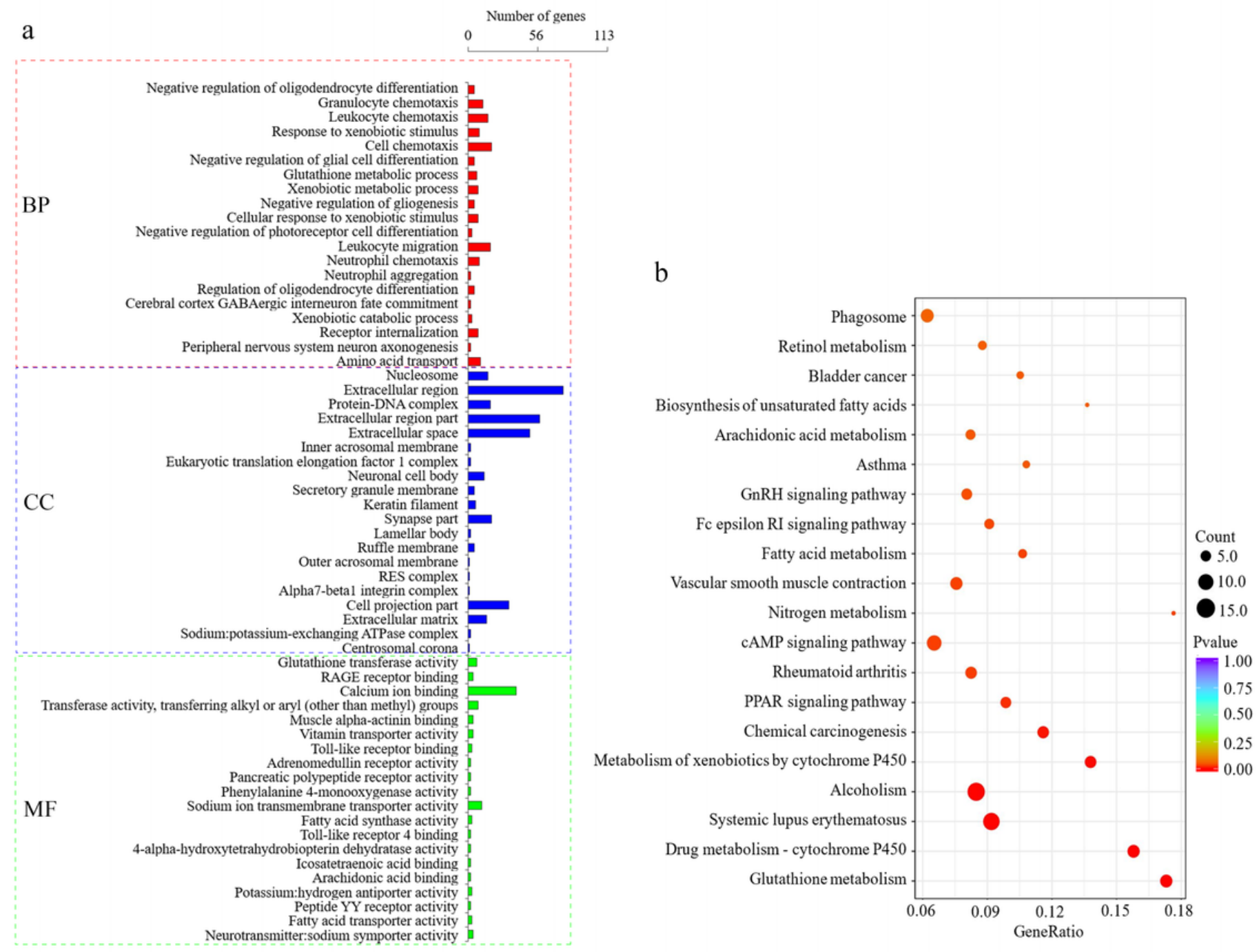

Figure 7

GO and KEGG enrichment analysis of DELs target genes predicted by co-expression. a: The first 20 significant GO items $(P<0.05)$. b: The first 20 KEGG enrichment results. 


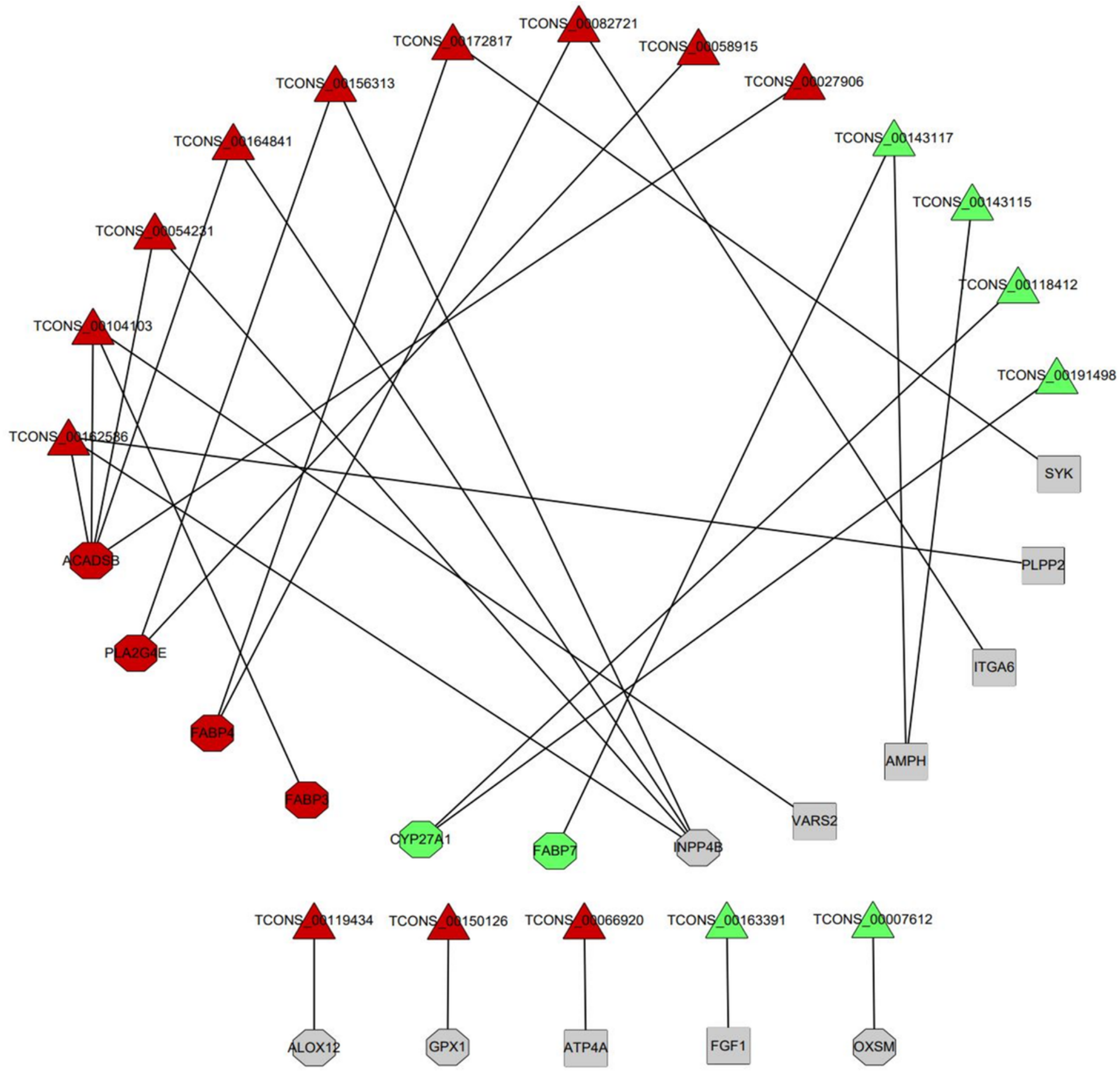

\section{Figure 8}

Regulatory relationship between DELs and target genes related to lipid metabolism. The triangle is the DELs, octagon is the target gene predicted by the DELs through the co-expression (trans), and the rectangle is the target gene predicted by the DELs through the co-localization (cis), red represents upregulation and green represents down-regulation, grey indicates that the expression difference is not significant. 


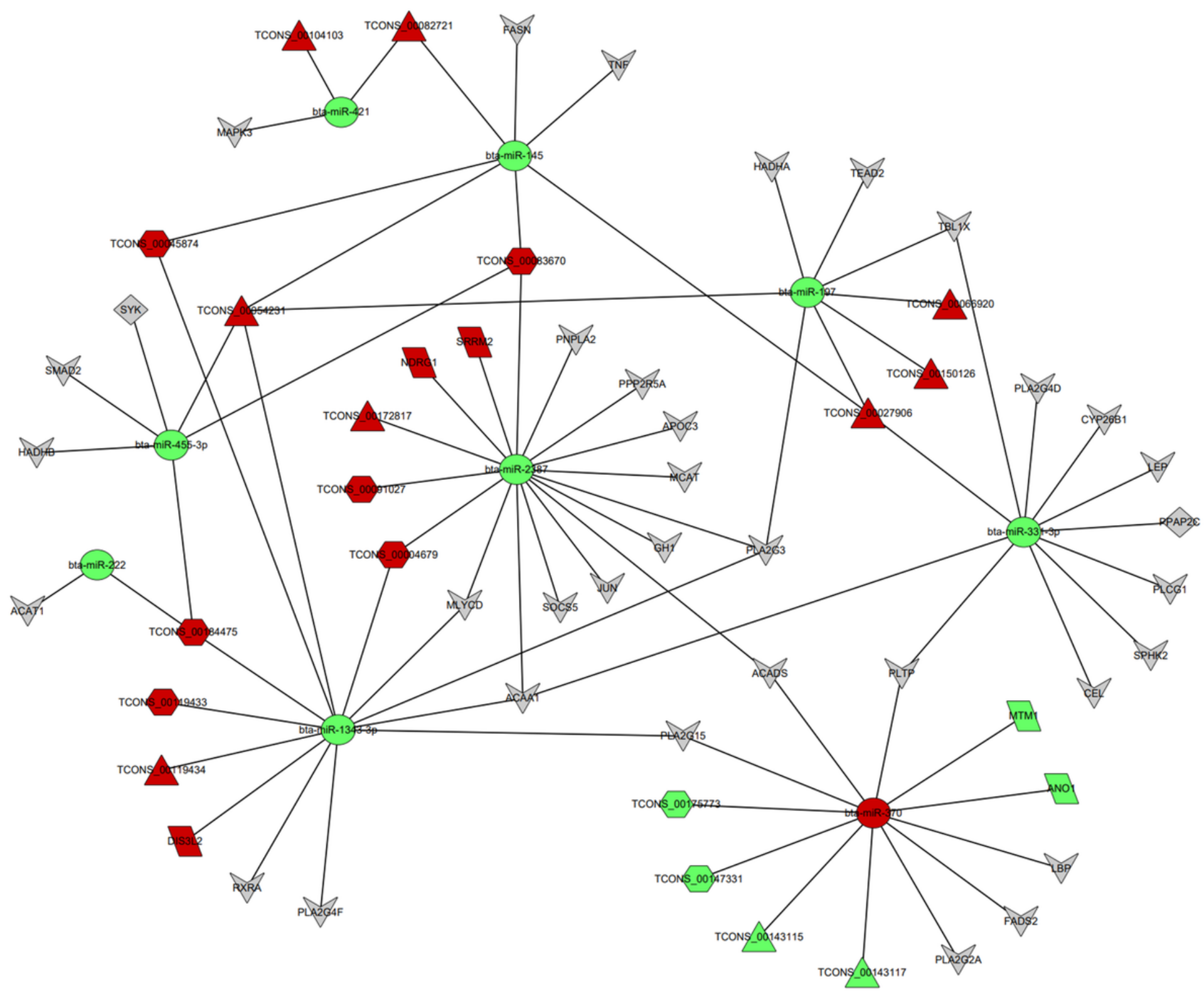

Figure 9

Regulatory network of DELs_ differential miRNAs_target genes. The triangle is lipid metabolism related DELs, hexagons are non lipid metabolism related DELs, circle represents differential miRNAs, arrow represents differential miRNAs target genes, regular quadrilateral represents DELs lipid metabolism related target genes, parallelogram represents DEGs, red represents upregulated expression and green represents downregulated expression, gray indicates that the expression difference is not significant. 


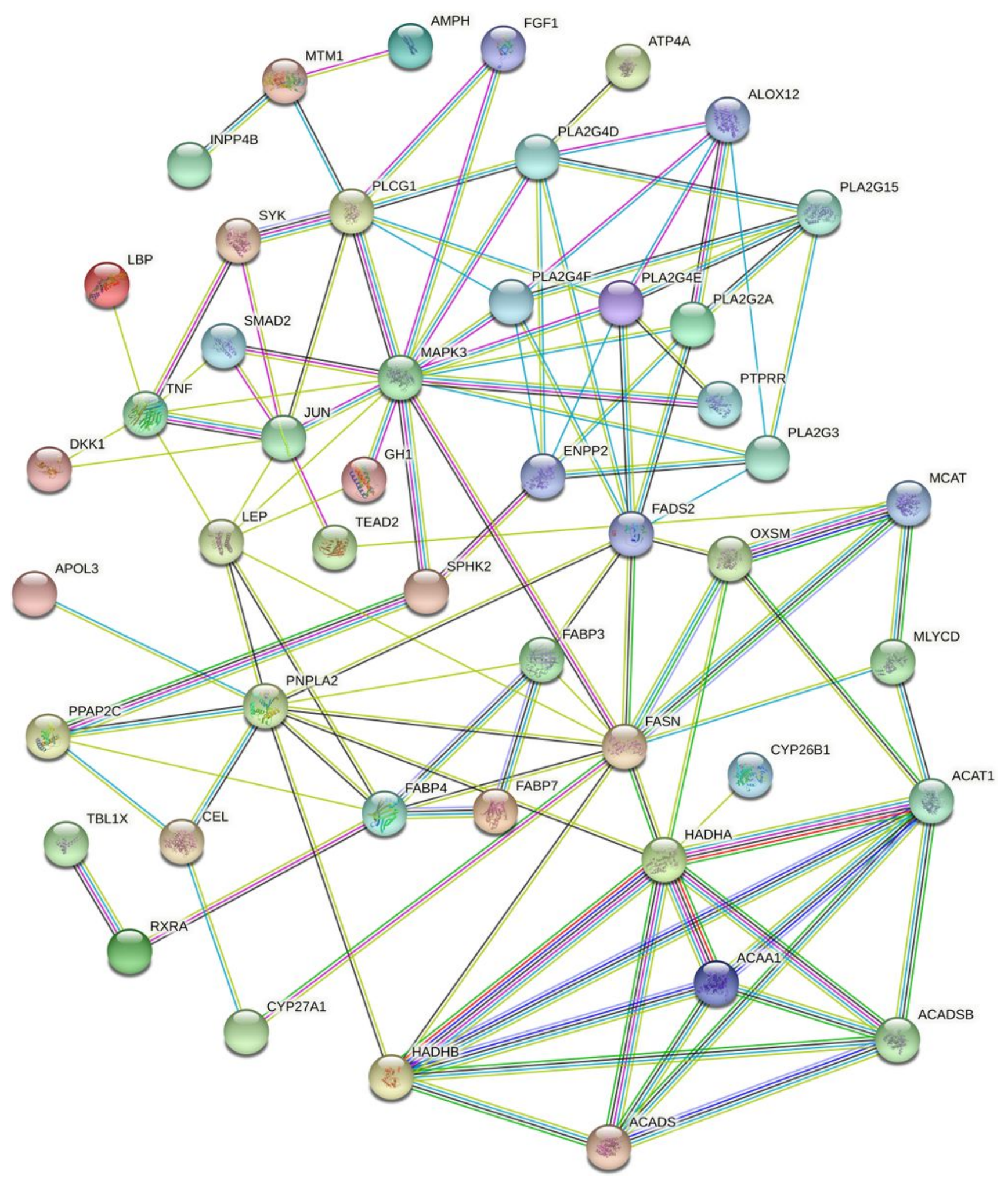

Figure 10

See the Supplemental Files section for the complete figure caption 

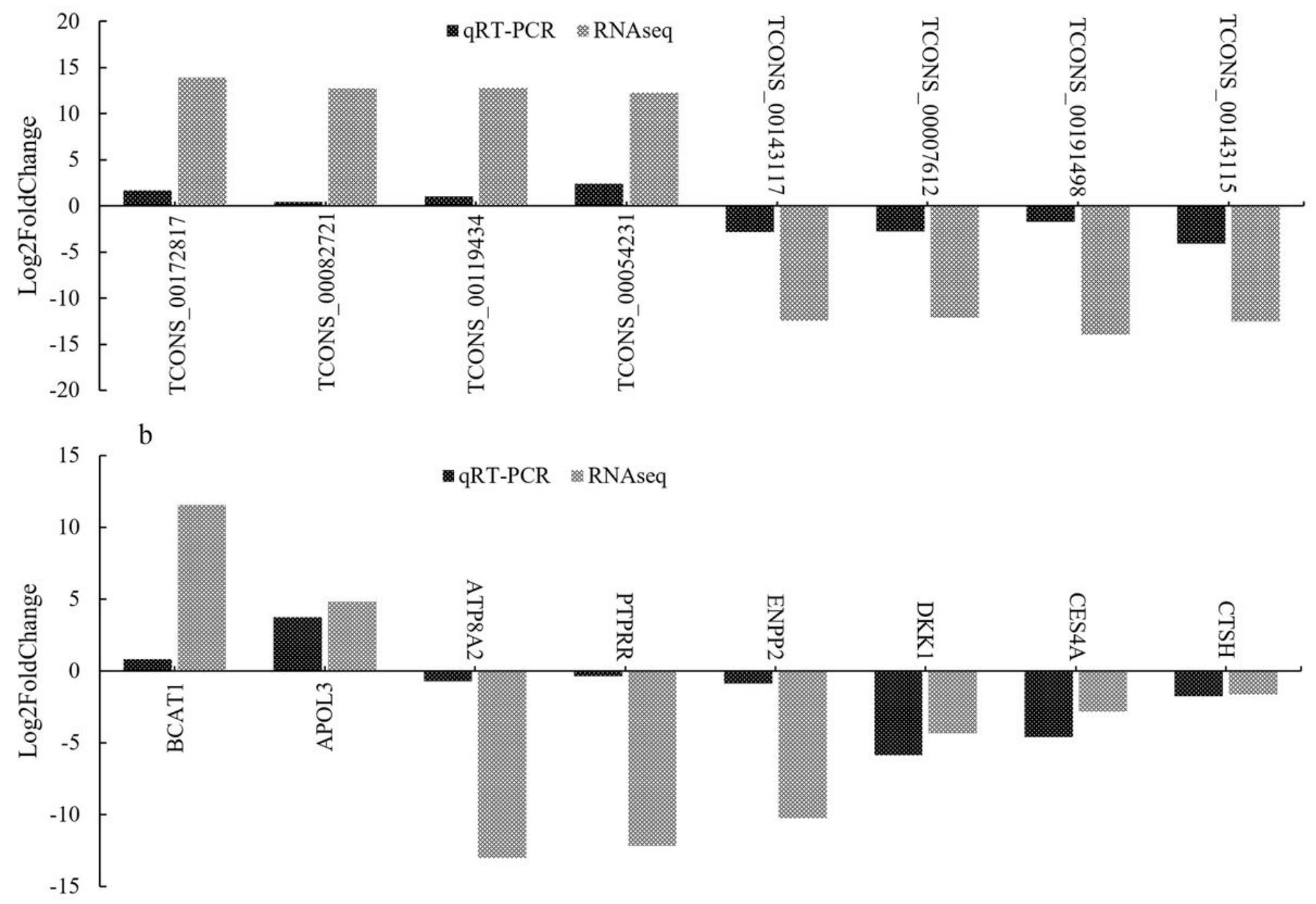

Figure 11

qRT-PCR verification of DEGs and DELs. a: The verification result of DELs. $b$ : The verification result of DEGs. log2FoldChange is logarithm of fold change with a base of 2 .

\section{Supplementary Files}

This is a list of supplementary files associated with this preprint. Click to download.

- SupplementaryTable.docx 